University of Wollongong

Research Online

Faculty of Engineering and Information

Faculty of Engineering and Information

Sciences - Papers: Part A

Sciences

$1-1-2014$

Asymmetric stochastic user equilibrium problem with elastic demand and link capacity constraints

Qiang Meng

National University of Singapore

Zhiyuan Liu

Monash University

Shuaian Wang

University of Wollongong, shuaian@uow.edu.au

Follow this and additional works at: https://ro.uow.edu.au/eispapers

Part of the Engineering Commons, and the Science and Technology Studies Commons

Research Online is the open access institutional repository for the University of Wollongong. For further information contact the UOW Library: research-pubs@uow.edu.au 


\title{
Asymmetric stochastic user equilibrium problem with elastic demand and link capacity constraints
}

\begin{abstract}
This paper focuses on model development and algorithm design for the general stochastic user equilibrium (SUE) problem with elastic demand, asymmetric link travel time functions and link capacity constraints. It first defines the generalised SUE conditions using generalised link travel time. An equivalent variational inequality $(\mathrm{VI})$ model for these generalised SUE conditions is then developed and it is rigorously proven to be monotone and uniform Lipschitzcontinuous. These two properties of the proposed VI model ensure the global convergence of the self-adaptive prediction-correction algorithm incorporating cost averaging method as a solution algorithm. Finally, a numerical example is utilised to assess the performance of the proposed VI model and solution algorithm.
\end{abstract}

\section{Keywords}

user, equilibrium, stochastic, problem, capacity, elastic, demand, constraints, asymmetric, link

\section{Disciplines}

Engineering | Science and Technology Studies

\section{Publication Details}

Meng, Q., Liu, Z. \& Wang, S. (2014). Asymmetric stochastic user equilibrium problem with elastic demand and link capacity constraints. Transportmetrica A: Transport Science, 10 (4), 304-326. 


\title{
ASYMMETRIC STOCHASTIC USER EQUILIBRIUM PROBLEM WITH ELASTIC DEMAND AND LINK CAPACITY CONSTRAINTS
}

\author{
Qiang Meng ${ }^{a}$, Zhiyuan Liu ${ }^{* a \& b}$, Shuaian Wang ${ }^{a \& c}$ \\ ${ }^{a}$ Department of Civil and Environmental Engineering, National University of Singapore, \\ Singapore 117576 \\ ${ }^{b}$ Institute of Transport Studies, Department of Civil Engineering, Monash University, Clayton, \\ Victoria 3800, Australia \\ ${ }^{c}$ School of Mathematics and Applied Statistics, University of Wollongong, Wollongong, NSW \\ 2522, Australia
}

\begin{abstract}
This paper focuses on model development and algorithm design for the general stochastic user equilibrium (SUE) problem with elastic demand, asymmetric link travel time functions and link capacity constraints. It first defines the generalized SUE conditions using generalized link travel time. An equivalent variational inequality (VI) model for these generalized SUE conditions is then developed and it is rigorously proven to be monotone and uniform Lipschitz-continuous. These two properties of the proposed VI model ensure the global convergence of the self-adaptive prediction-correction (PC) algorithm incorporating cost averaging (CA) method as a solution algorithm. Finally, a numerical example is utilized to assess the performance of proposed VI model and solution algorithm.
\end{abstract}

Key words: Stochastic user equilibrium; Link capacity constraints; Asymmetric link travel time functions; Elastic demand; Variational inequality

\section{INTRODUCTION}

Based on two widely accepted principles - deterministic user equilibrium (DUE) (Wardrop, 1952) and stochastic user equilibrium (SUE) (Daganzo and Sheffi, 1977), mathematical models and solution algorithms for the traffic assignment problems have been extensively developed and studied (e.g., Bell and Iida, 1997; Pravinvongvuth and Chen, 2005; Huang and Li, 2007; Bekhor et al., 2008; Cantarella and Cascetta, 2009; Chen et al., 2012; Zhou et al., 2012). These two principles both result from a behavioral assumption of network

\footnotetext{
${ }^{*}$ Corresponding author

Tel.: +61-3-99054951

Fax: +61-3-99054944

E-mail address: zhiyuan.liu@monash.edu (Z. Liu); ceemq@nus.edu.sg (Q. Meng); wangshuaian@gmail.com (S. Wang)
} 
users (drivers) that they would choose a path with minimum travel time between an origindestination (OD) pair. The DUE principle presumes that network users have accurate travel time information on the entire network, while the SUE principle more reasonably assumes that network users make their route choice based on their stochastic perceived travel times.

Three typical issues - elastic travel demand, asymmetric link travel time functions and link capacity constraints - are usually addressed to extend/modify the conventional DUE or SUE problem. Elasticity for travel demand between an OD pair is evidently a reasonable assumption because drivers would switch to a public transport mode if their travel time on a road network increases significantly. The asymmetric link travel time functions reflect interactions among link flows and Jacobian matrix of these link travel time functions is usually asymmetric. For brevity, the DUE/SUE problem with an asymmetric/symmetric Jacobian matrix of the link travel time functions is referred to as the asymmetric/symmetric DUE/SUE problem hereafter. As to the link capacity constraints, traffic flow on a link is restricted by a threshold from above due to its limited road space and/or some concerns of land transport authorities to mitigate traffic congestion or vehicle emission (Ferrari, 1997; Yang and Bell, 1997).

Although the asymmetric DUE problem with elastic demand and link capacity constraints has been well solved (e.g., Ferrari, 1997), it has long been an open question whether there exists a convergent and efficient algorithm for solving the asymmetric SUE problem with elastic demand and link capacity constraints. This paper therefore aims to seek for a solution to this unsolved yet important problem.

\subsection{Relevant Studies}

The asymmetric DUE problem with elastic demand is formulated as a variational inequality (VI) model in the literature (Smith, 1979; Dafermos, 1980). Simply incorporating link capacity constraints to the feasible solution set of this VI model gives a proper formulation for the asymmetric DUE problem with elastic demand and link capacity constraints. Any solution of this induced VI model fulfills the DUE conditions in terms of the generalized link travel time. Herein, generalized link travel time is defined as a summation of the actual travel time of a link and an optimal generalized Lagrangian multiplier associated with the link capacity constraint if any. For conciseness, the DUE conditions in terms of the generalized link travel time is called as the generalized DUE conditions. 
It should be pointed out that the asymmetric DUE problem with elastic demand and link capacity constraints can be equivalently transformed into a fixed demand case by adding a dummy link between each OD pair (Chapter 6, Sheffi, 1985). Subsequently, the diagonalization algorithm (Abdulaal and Leblanc, 1979; Dafermos 1982 \& 1983; Friesz et al., 1984) can be employed for solving the said VI model built for the asymmetric DUE problem with elastic demand and link capacity constraints. The diagonalization algorithm iteratively solves a sub-problem: the symmetric DUE problem with link capacity constraints. Four types of convergent methods can be adopted for solving this sub-problem: inner and outer penalty function methods (Inouye, 1987), Lagrangian multiplier method (Larsson and Patriksson, 1995), Lagrangian dual method (Larsson and Patriksson, 1999) and dual ascent method (Hearn and Lawphongpanich, 1990). Nie et al. (2004) numerically assessed performance of the inner penalty function method and Augmented Lagrangian multiplier method and concluded that both methods are able to find an acceptable solution, but their performances are substantially sensitive to the method used for solving their own sub-problem (a symmetric DUE problem without link capacity constraints). Therefore, the diagonalization algorithm incorporating any of these four algorithms can be employed to find a solution of the asymmetric DUE problem with link capacity constraints. Its convergence can be guaranteed under mild conditions (Florian and Spiess, 1982).

For the symmetric SUE problem with fixed demand, Daganzo (1982) and Sheffi and Powell (1982) contributed an unconstrained optimization model, respectively. They also demonstrated global convergence of the method of successive average (MSA) as a solution algorithm. Daganzo (1983) built a fixed-point model in terms of link flows for the asymmetric SUE problem with fixed demand and showed availability of the MSA. Inspired by Dagnazo (1983), Cantarella (1997) proposed two fixed-point models in terms of link flows and link costs respectively for the asymmetric SUE problem with elastic demand. He proved that the MSA updating link travel cost instead of link flow, called cost averaging (CA) method, is convergent for solving this SUE problem. Watling (1998) then discussed about the stability of solutions for the asymmetric SUE problem.

Other than the MSA and CA, some other algorithms are also observed from the literature for solving symmetric or asymmetric SUE problems. In the case of logit-based model, Chen and Alfa (1991) provided a heuristic line search procedure as an improvement of the predetermined step sizes used by MSA. This method, however, requires calculating the inverse of link-path incidence matrix, which is a tedious work in large-scale networks. 
Damberg et al. (1996) adopted the disaggregate simplicial decomposition algorithm based on a given route-set as a solution algorithm. A more efficient path-based algorithm was proposed by Bekhor and Toledo (2005). Other than these path-based algorithms, Maher (1998) developed a link-based algorithm with approximately step size with the same search direction as that of MSA. While, for the case of probit-based SUE, Maher and Hughes (1997) developed a stochastic assignment method (SAM), where a line search is conducted using Clark's approximation to calculate the value of objective function. Maher and Zhang (2000) presented another heuristic algorithm by using a quadratic function to approximate the objective function, which also enables a line-search technique. However, none of these studies for SUE problems has taken into account the link capacity constraints. It thus weakens their rationale when the achievements are used in practice.

Studies on the SUE problem with link capacity constraints are quite scarce, due to its complicated nature for formulation. Bell (1995) proposed an optimization model and a solution algorithm for the logit-based symmetric SUE problem with fixed demand and link capacity constraints. Meng et al. (2008) gave a linearly constrained convex minimization model and a convergent Lagrangian dual method for the general SUE problem with fixed demand, separable (symmetric) link travel time functions and link capacity constraints. Yet, the achievements of these two studies are not available for solving the asymmetric or elastic demand cases.

After the SUE principle proposed by Daganzo and Sheffi (1977), although there have been some extensions of the SUE principle (see, Mirchandani and Soroush, 1987; Watling, 2006; Patriksson, 2008; Baillon and Cominetti, 2008; Connors and Sumalee, 2009; Wei et al., 2012), the asymmetric SUE problem with elastic demand and link capacity constraints has not yet been solved. To the best of our knowledge, there is still no mathematical model that can contribute a global convergent solution algorithm for solving this problem. This hurdle comes from the fact that simply adding link capacity constraints to any standard SUE model cannot obtain an equivalent model for the corresponding SUE problem with link capacity constraints.

\subsection{Objective and Contributions}

The objective of this paper is to solve the aforementioned open question by developing a mathematical model and convergent algorithm for the asymmetric SUE problem with elastic demand and link capacity constraints. The generalized SUE conditions can be provided based 
on the generalized DUE conditions (Larsson and Patriksson, 1995). Any solution of a successful model should fulfill the generalized SUE conditions. Two questions are subsequently brought up: (a) the existence issue of solution to the generalized SUE conditions and (b) how to find a solution provided that such a solution does exist. To answer these two questions, this paper will develop a VI model defined on a non-empty, compact and convex set, and show that any solution of this VI model can fulfil the generalized SUE conditions. We will also rigorously demonstrate that the proposed VI model is monotone and uniform Lipschitz-continuous. These properties of the proposed VI model imply that it has at least one solution, i.e., there is a solution to the asymmetric SUE problem with elastic demand and link capacity constraints. More importantly, monotonicity and uniform Lipschitz-continuity of the proposed VI model enable us to employ the self-adaptive prediction-correction (PC) algorithm proposed by He and Liao (2002) as a convergent solution algorithm.

The remainder of this paper is organized as follows. Section 2 gives notation and assumptions as well as generalized SUE conditions for the asymmetric SUE problem with elastic demand and link capacity constraints. Section 3 develops a variational inequality (VI) model for the generalized SUE conditions and demonstrates its properties. Section 4 presents the self-adaptive PC algorithm incorporating CA method. Section 5 uses the Sioux-Falls network to assess the model and algorithm proposed in this study. Finally, conclusions are provided in Section 6.

\section{PROBLEM DESCRIPTION}

\subsection{Notation and Assumptions}

Given a transportation network represented by a strongly connected graph $G=(N, A)$ where $N$ and $A$ are the sets of nodes and links, respectively, let $W$ be the set of OD pairs and $R_{w}$ be the set of all the paths between OD pair $w \in W$. Travel demand between OD pair $w \in W$ is denoted by $q_{w}$ and all of these travel demands are grouped into vector $\mathbf{q}=\left(q_{w}, w \in W\right)^{\mathrm{T}}$. Let $f_{w k}$ be traffic flow on path $k \in R_{w}$ between OD pair $w \in W$ and all these path flows are grouped into vector $\mathbf{f}_{w}=\left(f_{w k}, k \in R_{w}\right)^{\mathrm{T}}$. The vector of all these path flows over the entire network is denoted by $\mathbf{f}=\left(\mathbf{f}_{w}, w \in W\right)^{\mathrm{T}}$. Traffic flow on link $a \in A$ is denoted by $v_{a}$ and all these link flows are grouped into vector $\mathbf{v}=\left(v_{a}, a \in A\right)^{\mathrm{T}}$. 
The feasible set of link flows, denoted by $\Theta$, is defined by the flow conservation equations, i.e.,

$$
\Theta=\{\mathbf{v} \mid \mathbf{v}=\Delta \mathbf{f}, \mathbf{q}=\Lambda \mathbf{f}, \mathbf{f} \geq 0\}
$$

where $\Delta=\left[\Delta_{w}, w \in W\right]$ is the link-path incidence matrix with sub-matrix $\Delta_{w}=\left[\delta_{a k}^{w}, a \in A, k \in R_{w}\right]$ in which $\delta_{a k}^{w}=1$ if path $k$ between OD pair $w \in W$ contains link $a$; and 0 otherwise. $\Lambda=\left[\delta_{k}^{w}, w \in W, k \in \cup_{w \in W} R_{w}\right]$ is the path-OD incidence matrix, where $\delta_{k}^{w}=1$ if path $k$ connects OD pair $w \in W$ and 0 otherwise.

It is assumed that each link $a \in \bar{A} \subseteq A$ has the link capacity constraint:

$$
v_{a} \leq H_{a}, a \in \bar{A}
$$

where $H_{a}$ is a given positive threshold on link $a \in \bar{A}$. Other than using physical capacity of traffic flow on the link, a smaller threshold is sometimes determined by traffic managers so as to mitigate traffic congestion and/or vehicle emission (Yang and Huang, 2005).

Travel time on each link $a \in A$ is assumed to be a function of all the link flows, denoted by $t_{a}(\mathbf{v})$, and let $\mathbf{t}(\mathbf{v})=\left(t_{a}(\mathbf{v}), a \in A\right)^{\mathrm{T}}$ denote the vector of link travel times. $\mathbf{t}(\mathbf{v})$ is continuously differentiable with positive definite Jacobian matrix $\nabla_{\mathbf{v}} \mathbf{t}(\mathbf{v})$ for any $\mathbf{v} \in \Theta$. The positive definiteness of Jacobian $\nabla_{\mathbf{v}} \mathbf{t}(\mathbf{v})$ implies the strictly monotonicity of link travel time function vector $\mathbf{t}(\mathbf{v})$ according to Theorem 5.4.3 of Ortega and Rheinboldt (1970), namely,

$$
\left(\mathbf{t}\left(\mathbf{v}^{\prime}\right)-\mathbf{t}\left(\mathbf{v}^{\prime \prime}\right)\right)^{\mathrm{T}}\left(\mathbf{v}^{\prime}-\mathbf{v}^{\prime \prime}\right)>0, \forall \mathbf{v}^{\prime}, \mathbf{v}^{\prime \prime} \in \Theta \text { and } \mathbf{v}^{\prime} \neq \mathbf{v}^{\prime \prime}
$$

In the context of SUE, the users make their route choice plans based on the perceived path travel time, denoted by $C_{w k}(\mathbf{f})$ :

$$
C_{w k}(\mathbf{f})=C_{w k}(\mathbf{f})+\zeta_{w k}, k \in R_{w}, w \in W
$$

where $c_{w k}(\mathbf{f})$ is the real path travel time, i.e., $c_{w k}(\mathbf{f})=\sum_{a \in A} t_{a}(\mathbf{v}) \delta_{a k}^{w}, k \in R_{w}, w \in W$. The perception error $\zeta_{w k}$ is a flow-independent random variable with zero mean and constant variance. The travel demand is assumed to be a continuously differentiable, non-increasing and bounded function with respect to the satisfaction $S_{w}\left(\mathbf{c}_{w}(\mathbf{f})\right)=E\left[\min \left(C_{w k}(\mathbf{f}), k \in R_{w}\right)\right]$ :

$$
q_{w}=D_{w}\left(S_{w}\left(\mathbf{c}_{w}(\mathbf{f})\right)\right) \leq \bar{q}_{w}, w \in W
$$

where parameter $\bar{q}_{w}$ is a given upper bound of travel demand between OD pair $w \in W$. It is assumed that travel demand between an OD pair will vanish when the travel time between this OD pair approaches infinity, i.e.,

$$
\lim _{x \rightarrow+\infty} D_{w}(x)=0, w \in W
$$




\subsection{Generalized SUE Conditions}

Cantarella (1997) put forward the following link-based fixed-point model for the asymmetric SUE problem with elastic demand: a link flow pattern $\mathbf{v}=\left(v_{a}, a \in A\right)^{\mathrm{T}} \in \Theta$ is a SUE solution if and only if

$$
v_{a}=\sum_{w \in W}\left[D_{w}\left(S_{w}\left(\Delta_{w}^{\mathrm{T}} \mathbf{t}(\mathbf{v})\right)\right) \times P_{w a}\left(\Delta_{w}^{\mathrm{T}} \mathbf{t}(\mathbf{v})\right)\right], a \in A
$$

where $P_{w a}\left(\Delta_{w}^{\mathrm{T}} \mathbf{t}(\mathbf{v})\right)$ is the link usage probability defined by

$$
P_{w a}\left(\Delta_{w}^{\mathrm{T}} \mathbf{t}(\mathbf{v})\right)=\sum_{k \in R_{w}} p_{w k}\left(\mathbf{c}_{w}(\mathbf{f})\right) \delta_{a k}^{w}, a \in A
$$

and $p_{w k}\left(\mathbf{c}_{w}(\mathbf{f})\right)$ is the probability that path $k$ between OD pair $w \in W$ is perceived as the shortest one among all the paths between the OD pair. Assuming the monotonicity of link travel time functions and demand functions, Cantarella (1997) demonstrated the existence and uniqueness of the SUE link flow solution. He also proposed a global convergent solution algorithm: cost averaging (CA) method.

A set of generalized SUE conditions was proposed by Meng et al. (2008) for the optimal solution of SUE problem with fixed demand, separate link travel time functions and link capacity constraints. These conditions can be extended to the case of elastic demand and asymmetric link travel time functions, which is the problem addressed in this paper: $\mathbf{v}=\left(v_{a}, a \in A\right)^{\mathrm{T}}$ is the SUE link flow solution of the addressed problem if and only if there exists a vector of Lagrangian multipliers corresponding to link capacity constraints, denoted by $\mathbf{u}^{*}=\left(u_{a}^{*}, a \in \bar{A}\right)^{\mathrm{T}}$, such that

$$
\begin{gathered}
v_{a}=\sum_{w \in W}\left[D_{w}\left(S_{w}\left(\Delta_{w}^{\mathrm{T}} \mathbf{t}(\mathbf{v})+\lambda_{w}\left(\mathbf{u}^{*}\right)\right)\right) \times P_{w a}\left(\Delta_{w}^{\mathrm{T}} \mathbf{t}(\mathbf{v})+\lambda_{w}\left(\mathbf{u}^{*}\right)\right)\right], a \in A \\
v_{a} \leq H_{a}, a \in \bar{A} \\
u_{a}^{*} \times\left(v_{a}-H_{a}\right)=0, a \in \bar{A} \\
u_{a}^{*} \geq 0, a \in \bar{A}
\end{gathered}
$$

where vector $\lambda_{w}\left(\mathbf{u}^{*}\right)$ is defined by

$$
\boldsymbol{\lambda}_{w}\left(\mathbf{u}^{*}\right)=\left(\lambda_{w k}\left(\mathbf{u}^{*}\right)=\sum_{a \in \bar{A}} u_{a}^{*} \delta_{a k}^{w}, k \in R_{w}\right)^{\mathrm{T}}, w \in W
$$

Based on the optimal Lagrangian multipliers $\mathbf{u}^{*}=\left(u_{a}^{*}, a \in \bar{A}\right)^{\mathrm{T}}$, the SUE problem with link capacity constraints is identical to the conventional SUE problem in terms of the generalized link travel time functions:

$$
\hat{t}_{a}\left(\mathbf{v}, \mathbf{u}^{*}\right)=\left\{\begin{array}{l}
t_{a}(\mathbf{v})+u_{a}^{*}, a \in \bar{A} \\
t_{a}(\mathbf{v}), a \in A \backslash \bar{A}
\end{array}, a \in A\right.
$$


Eqns. (10)-(12) imply that if there is no link capacity constraint on any link [这里是不是 应该是 $\mathrm{A}$ 而不是 $\mathrm{A}$ bar? ] $a \in \bar{A}$, then $u_{a}^{*}=0$. It should be noted that when the variance of each perceived error equals zero, the generalized SUE conditions becomes the generalized DUE conditions, where the path choice probability function is upper semi-continuous (Cantarella, 1997). The following two questions are revealed: (a) Does the optimal Lagrangian multiplier solution exist? (b) How to find such a solution if it really exists. To answer these two questions, the following section will give a variational inequality (VI) model for the generalized SUE conditions and Section 4 will introduce a global convergent solution algorithm for solving this VI model.

\section{MATHEMATICAL MODEL}

Before introducing the VI model, it is worthwhile to further highlight the challenges in modeling SUE problem with link capacity constraints. Formulation for any DUE problem with link capacity constraints can be easily handled by directly taking the link capacity constraints as side constraints to a model for the standard DUE problem with no link capacity constraint (e.g. Patriksson, 1994a; Larsson and Patriksson, 1999). However, when link capacity constraints are taken into consideration for the SUE problems, it considerably increases the challenges in formulation. For instance, directly adding link capacity constraints to the above fixed-point model proposed by Cantarella (1997) would undermine the existence of solution. Even for the SUE problem with separable link travel time functions, taking link capacity constraints to the optimization model proposed Daganzo (1982) or Maher and Zhang (2000) does not give us a suitable formulation, because the solution of such a model cannot satisfy the generalized SUE conditions.

The complementary slackness conditions, eqns. (10)-(12), can be regarded as a Nonlinear Complementarity Problem (NCP) of the vector of Lagrangian multipliers $\mathbf{u}=\left(u_{a}, a \in \bar{A}\right)^{\mathrm{T}}$. It is well known that when the feasible set of $\mathbf{u}=\left(u_{a}, a \in \bar{A}\right)^{\mathrm{T}}$ is the whole non-negative orthant, this NCP model is equivalent to the following VI model, denoted by $\operatorname{VI}\left(\boldsymbol{\Phi}, \mathfrak{R}_{+}^{|\bar{A}|}\right)$ :

$$
\boldsymbol{\Phi}\left(\mathbf{u}^{*}\right)^{\mathbf{T}}\left(\mathbf{u}-\mathbf{u}^{*}\right) \geq 0, \forall \mathbf{u} \in \mathfrak{R}_{+}^{|\bar{A}|}
$$

where $\mathfrak{R}_{+}^{|\bar{A}|}=\left\{\mathbf{u} \mid u_{a} \geq 0, a \in \bar{A}\right\}$ denotes the feasible set and $\boldsymbol{\Phi}(\mathbf{u})$ is a $|\bar{A}|$-dimensional vector function defined below:

$$
\boldsymbol{\Phi}(\mathbf{u})=\mathbf{H}-\mathbf{v}_{\bar{A}}(\mathbf{u})=\left(H_{a}-v_{a}(\mathbf{u}), a \in \bar{A}\right)^{\mathbf{T}}: \mathfrak{R}_{+}^{|\bar{A}|} \rightarrow \mathfrak{R}^{|\bar{A}|}
$$

where $\mathbf{H}=\left(H_{a}, a \in \bar{A}\right)^{\mathbf{T}}$ is the vector for all the thresholds for the link capacity constraints, and $\mathbf{v}_{\bar{A}}(\mathbf{u})$ is the sub-vector of SUE link flow solution $\mathbf{v}(\mathbf{u})=\left(\mathbf{v}_{\bar{A}}(\mathbf{u}), \mathbf{v}_{A \backslash \bar{A}}(\mathbf{u})\right)^{\mathrm{T}}$. Herein, 
$\mathbf{v}(\mathbf{u})$ is a link flow solution for the SUE problem with the following generalized link travel time functions (no link capacity constraint):

$$
\hat{t}_{a}(\mathbf{v}, \mathbf{u})=\left\{\begin{array}{l}
t_{a}(\mathbf{v})+u_{a}, a \in \bar{A} \\
t_{a}(\mathbf{v}), a \in A \backslash \bar{A}
\end{array}, \quad a \in A\right.
$$

In other words, vector $\mathbf{v}(\mathbf{u})$ is a solution of the fixed-point model with Lagrangian-multiplier vector $\mathbf{u}$ :

$$
v_{a}(\mathbf{u})=\sum_{w \in W}\left[D_{w}\left(S_{w}\left(\Delta_{w}^{\mathrm{T}} \hat{\mathbf{t}}(\mathbf{v}, \mathbf{u})\right)\right) \times P_{w a}\left(\Delta_{w}^{\mathrm{T}} \hat{\mathbf{t}}(\mathbf{v}, \mathbf{u})\right)\right], a \in A
$$

where $\hat{\mathbf{t}}(\mathbf{v}, \mathbf{u})=\left(\hat{t}_{a}(\mathbf{v}, \mathbf{u}), a \in A\right)^{\mathrm{T}}$ denotes a vector of all the generalized link travel time functions. $\mathbf{v}(\mathbf{u})$ is called as parametric SUE link flow vector in the remainder of this paper. In reality, sub-vector $\mathbf{v}_{\bar{A}}(\mathbf{u})$ is a collection of the parametric SUE link flows on those links with link capacity constraints.

Parametric SUE link flow vector $\mathbf{v}(\mathbf{u})$ is an implicit mapping of Lagrangian-multiplier vector $\mathbf{u}$. It can be easily shown that the generalized link travel time function vector $\hat{\mathbf{t}}(\mathbf{v}, \mathbf{u})$ is strictly monotone with respect to link flow vector $\mathbf{v}$ for any given non-negative Lagrangian-multiplier vector $\mathbf{u} \cdot \mathbf{v}(\mathbf{u})$ is therefore unique for any given $\mathbf{u}$ according to Theorem 2 of Cantarella (1997).

\subsection{Monotone and Continuous Properties of Vector Function $\Phi(\mathbf{u})$}

The existence of solution to VI model $V I\left(\boldsymbol{\Phi}, \mathfrak{R}_{+}^{|\bar{A}|}\right)$ as well as the global convergence of its solution algorithm depend on some fundamental properties of vector function $\boldsymbol{\Phi}(\mathbf{u})$. These properties mainly include monotonicity and continuity of vector function $\boldsymbol{\Phi}(\mathbf{u})$ (see, e.g. Patriksson, 1994b). Thus, we first rigorously show the following three important properties of vector function $\boldsymbol{\Phi}(\mathbf{u})$.

PROPOSITION 1. Vector function $\boldsymbol{\Phi}(\mathbf{u})$ is monotone on $\mathfrak{R}_{+}^{|\bar{A}|}$, namely,

$$
\left(\boldsymbol{\Phi}\left(\mathbf{u}^{\prime}\right)-\boldsymbol{\Phi}\left(\mathbf{u}^{\prime \prime}\right)\right)^{\mathrm{T}}\left(\mathbf{u}^{\prime}-\mathbf{u}^{\prime \prime}\right) \geq 0, \forall \mathbf{u}^{\prime}, \mathbf{u}^{\prime \prime} \in \mathfrak{R}_{+}^{|\bar{A}|}
$$

PROOF. For any two distinct non-negative Lagrangian-multiplier vectors $\mathbf{u}^{\prime}$ and $\mathbf{u}^{\prime \prime}$, let $\mathbf{v}\left(\mathbf{u}^{\prime}\right)$ and $\mathbf{v}\left(\mathbf{u}^{\prime \prime}\right)$ denote the corresponding SUE link flow solutions. Hence, there are two SUE path solutions $\mathbf{f}\left(\mathbf{u}^{\prime}\right)=\left(\mathbf{f}_{w}\left(\mathbf{u}^{\prime}\right), w \in W\right)^{\mathrm{T}}$ and $\mathbf{f}\left(\mathbf{u}^{\prime \prime}\right)=\left(\mathbf{f}_{w}\left(\mathbf{u}^{\prime \prime}\right), w \in W\right)^{\mathrm{T}}$ such that

$$
\begin{gathered}
\mathbf{v}\left(\mathbf{u}^{\prime}\right)=\Delta \mathbf{f}\left(\mathbf{u}^{\prime}\right) \\
\mathbf{v}\left(\mathbf{u}^{\prime \prime}\right)=\Delta \mathbf{f}\left(\mathbf{u}^{\prime \prime}\right) \\
\mathbf{f}_{w}\left(\mathbf{u}^{\prime}\right)=D_{w}\left(S_{w}\left(\hat{\mathbf{c}}_{w}^{\prime}\right)\right) \times \mathbf{p}_{w}\left(\hat{\mathbf{c}}_{w}^{\prime}\right), w \in W \\
\mathbf{f}_{w}\left(\mathbf{u}^{\prime \prime}\right)=D_{w}\left(S_{w}\left(\hat{\mathbf{c}}_{w}^{\prime \prime}\right)\right) \times \mathbf{p}_{w}\left(\hat{\mathbf{c}}_{w}^{\prime \prime}\right), w \in W
\end{gathered}
$$


where four vectors: $\hat{\mathbf{c}}_{w}^{\prime}=\Delta_{w}^{\mathrm{T}} \hat{\mathbf{t}}\left(\mathbf{v}\left(\mathbf{u}^{\prime}\right), \mathbf{u}^{\prime}\right), \hat{\mathbf{c}}_{w}^{\prime \prime}=\Delta_{w}^{\mathrm{T}} \hat{\mathbf{t}}\left(\mathbf{v}\left(\mathbf{u}^{\prime \prime}\right), \mathbf{u}^{\prime \prime}\right), \mathbf{p}_{w}\left(\hat{\mathbf{c}}^{\prime}\right)=\left(p_{w k}\left(\hat{\mathbf{c}}_{w}^{\prime}\right), k \in R_{w}\right)^{\mathrm{T}}$ and $\mathbf{p}_{w}\left(\hat{\mathbf{c}}^{\prime \prime}\right)=\left(p_{w k}\left(\hat{\mathbf{c}}_{w}^{\prime \prime}\right), k \in R_{w}\right)^{\mathrm{T}}$.

Since satisfaction function $S_{w}\left(\hat{\mathbf{c}}_{w}\right)$ is concave (Sheffi, 1985; Cantarella, 1997), it follows that

$$
\begin{aligned}
& S_{w}\left(\hat{\mathbf{c}}_{w}^{\prime}\right) \leq S_{w}\left(\hat{\mathbf{c}}_{w}^{\prime \prime}\right)+\left(\mathbf{p}_{w}\left(\hat{\mathbf{c}}_{w}^{\prime \prime}\right)\right)^{\mathrm{T}}\left(\hat{\mathbf{c}}_{w}^{\prime}-\hat{\mathbf{c}}_{w}^{\prime \prime}\right), w \in W \\
& S_{w}\left(\hat{\mathbf{c}}_{w}^{\prime \prime}\right) \leq S_{w}\left(\hat{\mathbf{c}}_{w}^{\prime}\right)+\left(\mathbf{p}_{w}\left(\hat{\mathbf{c}}_{w}^{\prime}\right)\right)^{\mathrm{T}}\left(\hat{\mathbf{c}}_{w}^{\prime \prime}-\hat{\mathbf{c}}_{w}^{\prime}\right), w \in W
\end{aligned}
$$

After multiplying both sides of eqns. (24) and (25) by $D_{w}\left(S_{w}\left(\hat{\mathbf{c}}_{w}^{\prime \prime}\right)\right)$ and $D_{w}\left(S_{w}\left(\hat{\mathbf{c}}_{w}^{\prime}\right)\right)$, respectively, we have

$$
\begin{aligned}
& D_{w}\left(S_{w}\left(\hat{\mathbf{c}}_{w}^{\prime \prime}\right)\right) \times\left(S_{w}\left(\hat{\mathbf{c}}_{w}^{\prime}\right)-S_{w}\left(\hat{\mathbf{c}}_{w}^{\prime \prime}\right)\right) \leq\left(\mathbf{f}_{w}\left(\mathbf{u}^{\prime \prime}\right)\right)^{\mathrm{T}}\left(\hat{\mathbf{c}}_{w}^{\prime}-\hat{\mathbf{c}}_{w}^{\prime \prime}\right), w \in W \\
& D_{w}\left(S_{w}\left(\hat{\mathbf{c}}_{w}^{\prime}\right)\right) \times\left(S_{w}\left(\hat{\mathbf{c}}_{w}^{\prime \prime}\right)-S_{w}\left(\hat{\mathbf{c}}_{w}^{\prime}\right)\right) \leq\left(\mathbf{f}_{w}\left(\mathbf{u}^{\prime}\right)\right)^{\mathrm{T}}\left(\hat{\mathbf{c}}_{w}^{\prime \prime}-\hat{\mathbf{c}}_{w}^{\prime}\right), w \in W
\end{aligned}
$$

Adding up eqn. (26) and eqn. (27) yields that

$$
\begin{gathered}
{\left[D_{w}\left(S_{w}\left(\hat{\mathbf{c}}_{w}^{\prime \prime}\right)\right)-D_{w}\left(S_{w}\left(\hat{\mathbf{c}}_{w}^{\prime}\right)\right)\right] \times\left[S_{w}\left(\hat{\mathbf{c}}_{w}^{\prime}\right)-S_{w}\left(\hat{\mathbf{c}}_{w}^{\prime \prime}\right)\right]} \\
\leq\left[\mathbf{f}_{w}\left(\mathbf{u}^{\prime \prime}\right)-\mathbf{f}_{w}\left(\mathbf{u}^{\prime}\right)\right]^{\mathrm{T}}\left(\hat{\mathbf{c}}_{w}^{\prime}-\hat{\mathbf{c}}_{w}^{\prime \prime}\right), w \in W
\end{gathered}
$$

namely,

$$
\begin{gathered}
{\left[D_{w}\left(S_{w}\left(\hat{\mathbf{c}}_{w}^{\prime}\right)\right)-D_{w}\left(S_{w}\left(\hat{\mathbf{c}}_{w}^{\prime \prime}\right)\right)\right] \times\left[S_{w}\left(\hat{\mathbf{c}}_{w}^{\prime}\right)-S_{w}\left(\hat{\mathbf{c}}_{w}^{\prime \prime}\right)\right]} \\
\geq\left[\mathbf{f}_{w}\left(\mathbf{u}^{\prime \prime}\right)-\mathbf{f}_{w}\left(\mathbf{u}^{\prime}\right)\right]^{\mathrm{T}}\left(\hat{\mathbf{c}}_{w}^{\prime}-\hat{\mathbf{c}}_{w}^{\prime \prime}\right), w \in W
\end{gathered}
$$

The monotonicity of OD demand functions implies that

$$
\left[D_{w}\left(S_{w}\left(\hat{\mathbf{c}}_{w}^{\prime}\right)\right)-D_{w}\left(S_{w}\left(\hat{\mathbf{c}}_{w}^{\prime \prime}\right)\right)\right] \times\left[S_{w}\left(\hat{\mathbf{c}}_{w}^{\prime}\right)-S_{w}\left(\hat{\mathbf{c}}_{w}^{\prime \prime}\right)\right] \leq 0, w \in W
$$

According to eqns. (29) and (30), we thus have:

$$
\left[\mathbf{f}_{w}\left(\mathbf{u}^{\prime}\right)-\mathbf{f}_{w}\left(\mathbf{u}^{\prime \prime}\right)\right]^{\mathrm{T}}\left(\hat{\mathbf{c}}_{w}^{\prime}-\hat{\mathbf{c}}_{w}^{\prime \prime}\right) \leq 0, w \in W
$$

Because $\hat{\mathbf{c}}_{w}^{\prime}=\Delta_{w}^{\mathrm{T}} \hat{\mathbf{t}}\left(\mathbf{v}\left(\mathbf{u}^{\prime}\right), \mathbf{u}^{\prime}\right)$ and $\hat{\mathbf{c}}_{w}^{\prime \prime}=\Delta_{w}^{\mathrm{T}} \hat{\mathbf{t}}\left(\mathbf{v}\left(\mathbf{u}^{\prime \prime}\right)\right.$, $\left.\mathbf{u}^{\prime \prime}\right)$, eqn. (31) can be rewritten by

$$
\left(\mathbf{f}_{w}\left(\mathbf{u}^{\prime}\right)-\mathbf{f}_{w}\left(\mathbf{u}^{\prime \prime}\right)\right)^{\mathrm{T}}\left[\Delta_{w}^{\mathrm{T}} \hat{\mathbf{t}}\left(\mathbf{v}\left(\mathbf{u}^{\prime}\right), \mathbf{u}^{\prime}\right)-\Delta_{w}^{\mathrm{T}} \hat{\mathbf{t}}\left(\mathbf{v}\left(\mathbf{u}^{\prime \prime}\right), \mathbf{u}^{\prime \prime}\right)\right] \leq 0, w \in W
$$

After rearranging the left hand side of eqn. (32), it follows that

$$
\begin{aligned}
& {\left[\mathbf{v}_{w}\left(\mathbf{u}^{\prime}\right)-\mathbf{v}_{w}\left(\mathbf{u}^{\prime \prime}\right)\right]^{\mathrm{T}}\left[\mathbf{t}\left(\mathbf{v}\left(\mathbf{u}^{\prime}\right)\right)-\mathbf{t}\left(\mathbf{v}\left(\mathbf{u}^{\prime \prime}\right)\right)\right]+\left[\mathbf{v}_{\bar{A}, w}\left(\mathbf{u}^{\prime}\right)-\mathbf{v}_{\bar{A}, w}\left(\mathbf{u}^{\prime \prime}\right)\right]^{\mathrm{T}}\left(\mathbf{u}^{\prime}-\mathbf{u}^{\prime \prime}\right)} \\
& \quad \leq 0, w \in W
\end{aligned}
$$

where the four link flow vectors associated with OD pair $w: \mathbf{v}_{w}\left(\mathbf{u}^{\prime}\right)=\left(v_{w a}\left(\mathbf{u}^{\prime}\right), a \in A\right)^{T}$, $\mathbf{v}_{w}\left(\mathbf{u}^{\prime \prime}\right)=\left(v_{w a}\left(\mathbf{u}^{\prime \prime}\right), a \in A\right)^{T}, \mathbf{v}_{w, \bar{A}}\left(\mathbf{u}^{\prime}\right)=\left(v_{w a}\left(\mathbf{u}^{\prime}\right), a \in \bar{A}\right)^{T}$, and $\mathbf{v}_{w, \bar{A}}\left(\mathbf{u}^{\prime \prime}\right)=\left(v_{w a}\left(\mathbf{u}^{\prime \prime}\right), a \in \bar{A}\right)^{T}$ with the elements:

$$
\begin{aligned}
& \mathbf{v}_{w a}\left(\mathbf{u}^{\prime}\right)=\sum_{k \in R_{w}} f_{w k}\left(\mathbf{u}^{\prime}\right) \delta_{a k}^{w}, a \in A \\
& \mathbf{v}_{w a}\left(\mathbf{u}^{\prime \prime}\right)=\sum_{k \in R_{w}} f_{w k}\left(\mathbf{u}^{\prime \prime}\right) \delta_{a k}^{w}, a \in A
\end{aligned}
$$

According to eqn. (33), we thus have 


$$
\begin{aligned}
& \sum_{w \in W}\left\{\left[\mathbf{v}_{w}\left(\mathbf{u}^{\prime}\right)-\mathbf{v}_{w}\left(\mathbf{u}^{\prime \prime}\right)\right]^{\mathrm{T}}\left[\mathbf{t}\left(\mathbf{v}\left(\mathbf{u}^{\prime}\right)\right)-\mathbf{t}\left(\mathbf{u}^{\prime \prime}\right)\right]+\left[\mathbf{v}_{w, \bar{A}}\left(\mathbf{u}^{\prime}\right)-\mathbf{v}_{w, \bar{A}}\left(\mathbf{u}^{\prime \prime}\right)\right]^{\mathrm{T}}\left(\mathbf{u}^{\prime}-\mathbf{u}^{\prime \prime}\right)\right\} \\
& \leq 0
\end{aligned}
$$

namely,

$$
\left[\mathbf{v}_{\bar{A}}\left(\mathbf{u}^{\prime}\right)-\mathbf{v}_{\bar{A}}\left(\mathbf{u}^{\prime \prime}\right)\right]^{\mathrm{T}}\left(\mathbf{u}^{\prime}-\mathbf{u}^{\prime \prime}\right) \leq-\left[\mathbf{v}\left(\mathbf{u}^{\prime}\right)-\mathbf{v}\left(\mathbf{u}^{\prime \prime}\right)\right]^{\mathrm{T}}\left[\mathbf{t}\left(\mathbf{v}\left(\mathbf{u}^{\prime}\right)\right)-\mathbf{t}\left(\mathbf{v}\left(\mathbf{u}^{\prime \prime}\right)\right)\right]
$$

The right hand side of eqn. (37) is non-negative since link travel time function vector $\mathbf{t}(\mathbf{v})$ is strictly monotone. Thus, if $\mathbf{v}_{\bar{A}}\left(\mathbf{u}^{\prime}\right) \neq \mathbf{v}_{\bar{A}}\left(\mathbf{u}^{\prime \prime}\right)$, it gives that

$$
\left[\mathbf{v}_{\bar{A}}\left(\mathbf{u}^{\prime}\right)-\mathbf{v}_{\bar{A}}\left(\mathbf{u}^{\prime \prime}\right)\right]^{\mathrm{T}}\left(\mathbf{u}^{\prime}-\mathbf{u}^{\prime \prime}\right)<0
$$

Otherwise, if $\mathbf{v}_{\bar{A}}\left(\mathbf{u}^{\prime}\right)=\mathbf{v}_{\bar{A}}\left(\mathbf{u}^{\prime \prime}\right)$, it follows that

$$
\left[\mathbf{v}_{\bar{A}}\left(\mathbf{u}^{\prime}\right)-\mathbf{v}_{\bar{A}}\left(\mathbf{u}^{\prime \prime}\right)\right]^{\mathrm{T}}\left(\mathbf{u}^{\prime}-\mathbf{u}^{\prime \prime}\right)=0
$$

According to eqns. (38) and (39), it is straightforward to see that

$$
\begin{aligned}
\left(\boldsymbol{\Phi}\left(\mathbf{u}^{\prime}\right)-\boldsymbol{\Phi}\left(\mathbf{u}^{\prime \prime}\right)\right)^{\mathrm{T}}\left(\mathbf{u}^{\prime}-\mathbf{u}^{\prime \prime}\right) & =\left(H-\mathbf{v}_{\bar{A}}\left(\mathbf{u}^{\prime}\right)-H+\mathbf{v}_{\bar{A}}\left(\mathbf{u}^{\prime \prime}\right)\right)^{\mathrm{T}}\left(\mathbf{u}^{\prime}-\mathbf{u}^{\prime \prime}\right) \\
& =-\left(\mathbf{v}_{\bar{A}}\left(\mathbf{u}^{\prime}\right)-\mathbf{v}_{\bar{A}}\left(\mathbf{u}^{\prime \prime}\right)\right)^{\mathrm{T}}\left(\mathbf{u}^{\prime}-\mathbf{u}^{\prime \prime}\right) \geq 0
\end{aligned}
$$

In other words, vector function $\boldsymbol{\Phi}(\mathbf{u})$ is monotone on $\mathfrak{R}_{+}^{|\bar{A}|}$.

PROPOSITION 2. Vector function $\boldsymbol{\Phi}(\mathbf{u})$ is continuously differentiable on $\mathfrak{R}_{+}^{|\bar{A}|}$.

PROOF. The implicit function theorem is used to prove this proposition. We first define the vector function $\mathbf{g}(\mathbf{v}, \mathbf{u})$ on $\Theta \times \mathfrak{R}_{+}^{|A|}$ as follows:

$$
\mathbf{g}(\mathbf{v}, \mathbf{u})=\left(g_{a}(\mathbf{v}, \mathbf{u}), a \in A\right)^{\mathrm{T}}
$$

where

$$
g_{a}(\mathbf{v}, \mathbf{u})=v_{a}-\sum_{w \in W} \sum_{k \in R_{w}}\left[D_{w}\left(S_{w}\left(\Delta_{w}^{\mathrm{T}} \hat{\mathbf{t}}(\mathbf{v}, \mathbf{u})\right)\right) \times p_{w k}\left(\Delta_{w}^{\mathrm{T}} \hat{\mathbf{t}}(\mathbf{v}, \mathbf{u})\right) \delta_{a k}^{w}\right], a \in A
$$

Let $\nabla_{\mathbf{v}} \mathbf{g}(\mathbf{v}, \mathbf{u})$ and $\nabla_{\mathbf{u}} \mathbf{g}(\mathbf{v}, \mathbf{u})$ be Jacobian matrices of vector function $\mathbf{g}(\mathbf{v}, \mathbf{u})$ with respect to vector $\mathbf{v}$ and $\mathbf{u}$, respectively. According to eqns. (41)-(42), these two Jacobian matrices have the explicit expressions:

$$
\begin{aligned}
\nabla_{\mathbf{v}} \mathbf{g}(\mathbf{v}, \mathbf{u})= & \mathbf{I}-\sum_{w \in W}\left\{\frac{\partial D_{w}(\mathbf{v}, \mathbf{u})}{\partial S_{w}} \times\left[\left(\Delta_{w} \mathbf{p}_{w}\right) \cdot\left(\Delta_{w} \mathbf{p}_{w}\right)^{\mathrm{T}}\right] \times \nabla_{\mathbf{v}} \hat{\mathbf{t}}(\mathbf{v}, \mathbf{u})\right\} \\
& -\sum_{w \in W}\left\{D_{w}(\mathbf{v}, \mathbf{u}) \times\left[\Delta_{w} \cdot \nabla_{c} \mathbf{p}_{w} \cdot\left(\Delta_{w}\right)^{\mathrm{T}}\right] \times \nabla_{\mathbf{v}} \hat{\mathbf{t}}(\mathbf{v}, \mathbf{u})\right\} \\
\nabla_{\mathbf{u}} \mathbf{g}(\mathbf{v}, \mathbf{u})= & -\sum_{w \in W}\left\{\frac{\partial D_{w}(\mathbf{v}, \mathbf{u})}{\partial S_{w}} \times\left[\left(\Delta_{w} \mathbf{p}_{w}\right) \times\left(\Delta_{w} \mathbf{p}_{w}\right)^{\mathrm{T}}\right] \times \nabla_{\mathbf{u}} \hat{\mathbf{t}}(\mathbf{v}, \mathbf{u})\right\} \\
& -\sum_{w \in W}\left[D_{w}(\mathbf{v}, \mathbf{u}) \times\left[\Delta_{w} \cdot \nabla_{\hat{c}} \mathbf{p}_{w} \cdot\left(\Delta_{w}\right)^{\mathrm{T}}\right] \times \nabla_{\mathbf{u}} \hat{\mathbf{t}}(\mathbf{v}, \mathbf{u})\right]
\end{aligned}
$$


where $\mathbf{p}_{w}, S_{w}$, and $D_{w}(\mathbf{v}, \mathbf{u})$ are abbreviations of $\mathbf{p}_{w}=\left(p_{w k}\left(\Delta_{w}^{T} \hat{\mathbf{t}}(\mathbf{v}, \mathbf{u})\right), k \in R_{w}\right)^{\mathrm{T}}$, $S_{w}\left(\Delta_{w}^{T} \hat{\mathbf{t}}(\mathbf{v}, \mathbf{u})\right)$ and $D_{w}\left(S_{w}\left(\Delta_{w}^{T} \hat{\mathbf{t}}(\mathbf{v}, \mathbf{u})\right)\right)$, respectively. It should be noted that derivation of eqns. (43) and (44) uses the following property of satisfaction (Sheffi, 1985):

$$
\frac{\partial S_{w}\left(\hat{\mathbf{c}}_{w}(\mathbf{f})\right)}{\partial\left(\hat{c}_{w k}(\mathbf{f})\right)}=p_{w k}\left(\hat{\mathbf{c}}_{w}(\mathbf{f})\right), k \in R_{w}, w \in W
$$

For any given $\mathbf{u}_{0} \in \mathfrak{R}_{+}^{|\bar{A}|}$, an unique $\mathbf{v}_{0}=\mathbf{v}\left(\mathbf{u}_{0}\right)$ can be obtained. We now proceed to demonstrate that $\nabla_{\mathbf{v}} \mathbf{g}\left(\mathbf{v}_{0}, \mathbf{u}_{0}\right)$ is non-singular. It is readily to verify that

$$
\nabla_{\mathbf{v}} \hat{\mathbf{t}}\left(\mathbf{v}_{0}, \mathbf{u}_{0}\right)=\nabla_{\mathbf{v}} \mathbf{t}\left(\mathbf{v}_{0}\right)
$$

As Jacobian matrix $\nabla_{\mathbf{v}} \mathbf{t}\left(\mathbf{v}_{0}\right)$ is positive definite, $\nabla_{\mathbf{v}} \mathbf{t}\left(\mathbf{v}_{0}\right)$ is thereby non-singular and its inverse $\left[\nabla_{\mathbf{v}} \mathbf{t}\left(\mathbf{v}_{0}\right)\right]^{-1}$ is also positive definite. According to eqn. (43), Jacobian matrix $\nabla_{\mathbf{v}} \mathbf{g}(\mathbf{v}, \mathbf{u})$ at $\left(\mathbf{v}_{0}, \mathbf{u}_{0}\right)$ can be rewritten as follows:

$$
\begin{aligned}
\nabla_{\mathbf{v}} \mathbf{g}\left(\mathbf{v}_{0}, \mathbf{u}_{0}\right)=\{ & {\left[\nabla_{\mathbf{v}} \mathbf{t}\left(\mathbf{v}_{0}\right)\right]^{-1}-\sum_{w \in W}\left\{\frac{\partial D_{w}\left(\mathbf{v}_{0}, \mathbf{u}_{0}\right)}{\partial S_{w}} \times\left[\left(\Delta_{w} \mathbf{p}_{w}\right) \cdot\left(\Delta_{w} \mathbf{p}_{w}\right)^{\mathrm{T}}\right]\right\} } \\
& \left.-\sum_{w \in W}\left\{D_{w}\left(\mathbf{v}_{0}, \mathbf{u}_{0}\right) \times\left[\Delta_{w} \cdot \nabla_{c} \mathbf{p}_{w} \cdot\left(\Delta_{w}\right)^{\mathrm{T}}\right]\right\}\right\} \times \nabla_{\mathbf{v}} \mathbf{t}\left(\mathbf{v}_{0}\right)
\end{aligned}
$$

There are three terms in the brace of the right hand side of eqn. (47). The first term $\left[\nabla_{\mathbf{v}} \mathbf{t}\left(\mathbf{v}_{0}\right)\right]^{-1}$ is positive definite. The second term is positive semi-definite because $\frac{\partial D_{w}\left(\mathbf{v}_{0}, \mathbf{u}_{0}\right)}{\partial S_{w}} \leq 0$ and $\left[\left(\Delta_{w} \mathbf{p}_{w}\right) \cdot\left(\Delta_{w} \mathbf{p}_{w}\right)^{\mathrm{T}}\right]$ is positive semi-definite. Since $\left[\Delta_{w} \cdot \nabla_{\hat{c}} \mathbf{p}_{w} \cdot\left(\Delta_{w}\right)^{\mathrm{T}}\right]$ is a negative semi-definite matrix (pp. 320, Sheffi, 1985) and $D_{w}\left(\mathbf{v}_{0}, \mathbf{u}_{0}\right) \geq 0$, the third term is thus also positive semi-definite. Hence, the whole part in the brace of the right hand side of eqn. (47) is a positive definite matrix. In other words, $\nabla_{\mathbf{v}} \mathbf{g}\left(\mathbf{v}_{0}, \mathbf{u}_{0}\right)$ equals to a positive definite matrix multiplied by a non-singular matrix. Therefore, $\nabla_{\mathbf{v}} \mathbf{g}\left(\mathbf{v}_{0}, \mathbf{u}_{0}\right)$ is non-singular.

According to eqns. (43)-(44), it can be seen that $\nabla_{\mathbf{v}} \mathbf{g}(\mathbf{v}, \mathbf{u})$ and $\nabla_{\mathbf{u}} \mathbf{g}(\mathbf{v}, \mathbf{u})$ both are continuous with respect to $(\mathbf{v}, \mathbf{u})$. As $\mathbf{v}\left(\mathbf{u}_{0}\right)$ is the parametric SUE link flow vector, i.e., a solution to the fixed-point model (18), it follows that

$$
\mathbf{g}\left(\mathbf{v}_{0}, \mathbf{u}_{0}\right)=\mathbf{0}
$$

Since $\nabla_{\mathbf{v}} \mathbf{g}\left(\mathbf{v}_{0}, \mathbf{u}_{0}\right)$ is a non-singular matrix, $\mathbf{v}(\mathbf{u})$ is continuously differentiable in a neighborhood of $\mathbf{u}_{0}$ according to the implicit function theorem (Theorem 5.2.4, Ortega and Rheinboldt, 1970) and it has the gradient:

$$
\nabla \mathbf{v}(\mathbf{u})=-\left[\nabla_{\mathbf{v}} \mathbf{g}(\mathbf{v}, \mathbf{u})\right]^{-1} \nabla_{\mathbf{u}} \mathbf{g}(\mathbf{v}, \mathbf{u})
$$


As $\mathbf{v}(\mathbf{u})$ is continuously differentiable on $\mathfrak{R}_{+}^{|\bar{A}|}, \boldsymbol{\Phi}(\mathbf{u})$ is thus continuously differentiable on $\mathfrak{R}_{+}^{|\bar{A}|}$ according to its definition shown in eqn. (16).

Since vector function $\mathbf{v}(\mathbf{u})$ is continuously differentiable on $\mathfrak{R}_{+}^{|\bar{A}|}$, its Jacobian matrix $\nabla_{\mathbf{u}} \boldsymbol{\Phi}(\mathbf{u})$ is thus continuous on $\mathfrak{R}_{+}^{|\bar{A}|}$. The 2-norm of $\nabla_{\mathbf{u}} \mathbf{v}(\mathbf{u})$ is therefore bounded from above over any non-empty and compact set denoted by $\Psi$, namely, there is a positive constant $L$ such that

$$
\left\|\nabla_{\mathbf{u}} \mathbf{v}(\mathbf{u})\right\|_{2} \leq L, \forall \mathbf{u} \in \Psi
$$

According to Theorem 3.2.4 of Ortega and Rheinboldt (1970) (mean-value theorem), it can be seen that

$$
\left\|\boldsymbol{\Phi}\left(\mathbf{u}^{\prime}\right)-\boldsymbol{\Phi}\left(\mathbf{u}^{\prime \prime}\right)\right\|_{2}=\left\|\mathbf{v}\left(\mathbf{u}^{\prime}\right)-\mathbf{v}\left(\mathbf{u}^{\prime \prime}\right)\right\|_{2} \leq L\left\|\mathbf{u}^{\prime}-\mathbf{u}^{\prime \prime}\right\|, \forall \mathbf{u}^{\prime}, \mathbf{u}^{\prime \prime} \in \Psi
$$

In other words, we have the following proposition.

PROPOSITION 3. Vector function $\mathbf{\Phi}(\mathbf{u})$ is uniform Lipschitz-continuous on any non-empty and compact set in $\mathfrak{R}_{+}^{|\bar{A}|}$.

\subsection{A Restricted Variational Inequality Model}

Though we have demonstrated three important properties of vector function $\boldsymbol{\Phi}(\mathbf{u})$, it is still quite difficult to show the existence of a solution to VI model $V I\left(\boldsymbol{\Phi}, \mathfrak{R}_{+}^{|\bar{A}|}\right)$ by means of some existing sufficient conditions; for instance, the coercivity condition (Facchinei and Pang, 2003). It is well known that a VI model has at least one solution if it is continuous over a compact set (Corollary 2.25, Facchinei and Pang, 2003). However, VI model VI $\left(\boldsymbol{\Phi}, \mathfrak{R}_{+}^{|\bar{A}|}\right)$ does not fulfill the compactness condition because $\mathfrak{R}_{+}^{|\bar{A}|}$ is an unbounded set. We thus intend

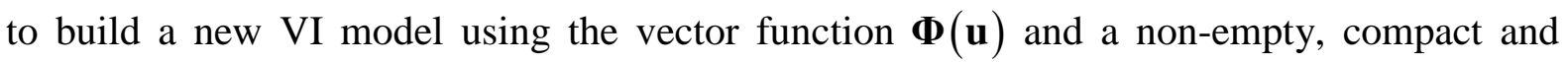
convex set.

According to the assumptions of OD demand functions and $\min _{a \in \bar{A}}\left\{H_{a}\right\}>0$, it can be seen that there is a positive number $M_{1}$ such that for any number $K \geq M_{1}$ we have

$$
D_{w}\left(\gamma_{1} K\right) \leq \gamma_{2} \times \min _{a \in \bar{A}}\left\{H_{a}\right\}, \forall w \in W
$$

where $\gamma_{1}, \gamma_{2} \in(0,1)$ are two given parameters, say $\gamma_{1}=0.5$ and $\gamma_{2}=0.9$. Since multivariate error variable $\zeta_{w}=\left(\zeta_{w k}, k \in R_{w}\right)^{\mathrm{T}}$ for OD pair $w \in W$ has a strictly positive and continuously differentiable probability density function, we can find a positive number $M_{2}$ such that for any number $K \geq M_{2}$ we have 


$$
\sum_{\substack{k \in R_{w} \\ k \neq l}} \operatorname{Pr}\left(\zeta_{w k}-\zeta_{w l} \leq \sum_{a \in A} t_{a}\left(\mathbf{v}^{\max }\right)-\left(1-\gamma_{1}\right) K\right) \leq \frac{\gamma_{2} \times \min _{a \in \bar{A}}\left\{H_{a}\right\}}{\left(|W| \times \bar{q}_{w}\right)}, \forall l \in R_{w}, \forall w \in W
$$

where $|W|$ is the number of OD pairs and vector $\mathbf{v}^{\max }=\left(v_{a}^{\max }=\sum_{w \in W} \bar{q}_{w}, a \in A\right)^{\mathrm{T}}$. By taking $M=\max \left(M_{1}, M_{2}\right)>0$, we construct a non-empty and compact convex set $\Omega \subset \mathfrak{R}_{+}^{|\bar{A}|}$ as follows:

$$
\Omega=\left\{\mathbf{u} \mid 0 \leq u_{a} \leq M=\max \left(M_{1}, M_{2}\right), a \in \bar{A}\right\}
$$

Accordingly, we define a restricted VI model on set $\Omega$ using vector function $\boldsymbol{\Phi}(\mathbf{u})$, denoted by $V I(\boldsymbol{\Phi}, \Omega)$ : Find a vector $\mathbf{u}^{*} \in \Omega$ such that

$$
\boldsymbol{\Phi}\left(\mathbf{u}^{*}\right)^{\mathbf{T}}\left(\mathbf{u}-\mathbf{u}^{*}\right) \geq 0, \forall \mathbf{u} \in \Omega
$$

As vector function $\boldsymbol{\Phi}(\mathbf{u})$ is continuous and $\Omega$ is a non-empty, convex and compact set, thus the restricted model $V I(\boldsymbol{\Phi}, \Omega)$ has at least one solution according to Corollary 2.25 of Facchinei and Pang (2003).

However, equivalence between this new VI model $V I(\boldsymbol{\Phi}, \Omega)$ and the generalized SUE conditions is not clear. We hence rigorously prove this equivalence condition, which is concluded in Proposition 4.

PROPOSITION 4. $\mathbf{u}^{*}=\left(u_{a}^{*}, a \in \bar{A}\right)$ is a solution of $V I(\boldsymbol{\Phi}, \Omega)$ if and only if $\mathbf{u}^{*}$ and $\mathbf{v}\left(\mathbf{u}^{*}\right)$ fulfill the generalized SUE conditions (9)-(12).

PROOF.

\section{Necessary condition}

Suppose that $\mathbf{u}^{*}=\left(u_{a}^{*}, a \in \bar{A}\right)$ is a solution of $V I(\Phi, \Omega)$ and we now show that $\mathbf{u}^{*}=\left(u_{a}^{*}, a \in \bar{A}\right)$ and $\mathbf{v}\left(\mathbf{u}^{*}\right)$ fulfills the generalized SUE conditions.

We first demonstrate that $u_{a}^{*}<M, \forall a \in \bar{A}$ by using an apagogical approach as follows. Assume that there is at least one link $b \in \bar{A}$ with $u_{b}^{*}=M$. Then, define a specific feasible vector $\mathbf{u}^{\prime} \in \Omega$ :

$$
\mathbf{u}^{\prime}=\left(u_{a}^{\prime}=u_{a}^{*}, a \in \bar{A} \backslash\{b\}, u_{b}^{\prime}=0.5 M\right)^{\mathrm{T}}
$$

After substituting $\mathbf{u}$ in the VI model $\operatorname{VI}(\boldsymbol{\Phi}, \Omega)$ with vector $\mathbf{u}^{\prime}$, it follows that

$$
\left(H_{b}-v_{b}\left(\mathbf{u}^{*}\right)\right) \times(0.5 M-M) \geq 0
$$

namely,

$$
v_{b}\left(\mathbf{u}^{*}\right) \geq H_{b}
$$

We proceed to show that $v_{b}\left(\mathbf{u}^{*}\right)<H_{b}$ by analyzing the following two cases:

Case 1: Suppose there is at least one path between O-D pair $w \in W$ not passing through the particular link $b$, say $k_{0} \in R_{w}$, with 


$$
\lambda_{w k_{0}}\left(\mathbf{u}^{*}\right)=\sum_{a \in A} u_{a}^{*} \delta_{a k}^{w} \leq \gamma_{1} M
$$

Let $R_{w b} \subset R_{w}$ be the set of all paths between OD pair $w \in W$ using link $b$ and $v_{w b}\left(\mathbf{u}^{*}\right)$ be the sum of traffic flow of all the paths between OD $w \in W$ pair passing though the particular link $b$, namely:

$$
v_{w b}\left(\mathbf{u}^{*}\right)=\sum_{k \in R_{w b}}\left[D_{w}\left(S_{w}\left(\Delta_{w}^{\mathrm{T}} \hat{\mathbf{t}}\left(\mathbf{v}\left(\mathbf{u}^{*}\right), \mathbf{u}^{*}\right)\right)\right) \times p_{w k}\left(\mathbf{c}_{w}\left(\mathbf{f}\left(\mathbf{u}^{*}\right)\right)+\boldsymbol{\lambda}_{w}\left(\mathbf{u}^{*}\right)\right)\right]
$$

We thus have

$$
v_{b}\left(\mathbf{u}^{*}\right)=\sum_{w \in W} v_{w b}\left(\mathbf{u}^{*}\right)
$$

According the assumption that OD demand function for OD pair $w \in W$ has the upper bound $\bar{q}_{w}$ and eqn. (59), it follows that

$$
\begin{aligned}
v_{w b}\left(\mathbf{u}^{*}\right)= & \sum_{k \in R_{w b}}\left[D_{w}\left(S_{w}\left(\Delta_{w}^{\mathrm{T}} \hat{\mathbf{t}}\left(\mathbf{v}\left(\mathbf{u}^{*}\right), \mathbf{u}^{*}\right)\right)\right) \times p_{w k}\left(\mathbf{c}_{w}\left(\mathbf{f}\left(\mathbf{u}^{*}\right)\right)+\lambda_{w}\left(\mathbf{u}^{*}\right)\right)\right] \\
& \leq \bar{q}_{w} \sum_{k \in R_{w b}} p_{w k}\left(\mathbf{c}_{w}\left(\mathbf{f}\left(\mathbf{u}^{*}\right)\right)+\boldsymbol{\lambda}_{w}\left(\mathbf{u}^{*}\right)\right)
\end{aligned}
$$

As the path choice probability

$$
\begin{aligned}
p_{w k}\left(\mathbf{c}_{w}\left(\mathbf{f}\left(\mathbf{u}^{*}\right)\right)+\lambda_{w}\left(\mathbf{u}^{*}\right)\right)= & \operatorname{Pr}\left(c_{w k}\left(\mathbf{f}\left(\mathbf{u}^{*}\right)\right)+\lambda_{w k}\left(\mathbf{u}^{*}\right)+\zeta_{w k} \leq\right. \\
& \left.c_{w l}\left(\mathbf{f}\left(\mathbf{u}^{*}\right)\right)+\lambda_{w l}\left(\mathbf{u}^{*}\right)+\zeta_{w l}, \forall l \in R_{w} \text { and } l \neq k\right), \forall k \in R_{w b}
\end{aligned}
$$

In the right hand side of eqn. (62), in the bracket, the perceived travel time on path $k \in R_{w}$ must be less than or equal to that on each of all the other paths between OD $w \in W$, including the particular path $k_{0} \in R_{w}$. We thus have

$$
\begin{aligned}
& p_{w k}\left(\mathbf{c}_{w}\left(\mathbf{f}\left(\mathbf{u}^{*}\right)\right)+\lambda_{w}\left(\mathbf{u}^{*}\right)\right) \leq \\
& \operatorname{Pr}\left(\zeta_{w k}-\zeta_{w k_{0}} \leq \sum_{a \in A} t_{a}\left(\mathbf{v}\left(\mathbf{u}^{*}\right)\right) \delta_{a k_{0}}^{w}+\lambda_{w k_{0}}\left(\mathbf{u}^{*}\right)-\sum_{a \in A} t_{a}\left(\mathbf{v}\left(\mathbf{u}^{*}\right)\right) \delta_{a k}^{w}-\lambda_{w k}\left(\mathbf{u}^{*}\right)\right), \forall k \in R_{w b}
\end{aligned}
$$

For any path $k \in R_{w b}$, it can seen that

$$
\begin{aligned}
& \sum_{a \in A} t_{a}\left(\mathbf{v}\left(\mathbf{u}^{*}\right)\right) \delta_{a k_{0}}^{w}+\lambda_{w k_{0}}\left(\mathbf{u}^{*}\right)-\left[\sum_{a \in A} t_{a}\left(\mathbf{v}\left(\mathbf{u}^{*}\right)\right) \delta_{a k}^{w}+\lambda_{w k}\left(\mathbf{u}^{*}\right)\right] \\
& \leq \sum_{a \in A} t_{a}\left(\mathbf{v}^{\max }\right)+\lambda_{w k_{0}}\left(\mathbf{u}^{*}\right)-\lambda_{w k}\left(\mathbf{u}^{*}\right) \\
& =\sum_{a \in A} t_{a}\left(\mathbf{v}^{\max }\right)+\lambda_{w k_{0}}\left(\mathbf{u}^{*}\right)-\left(u_{b}^{*}+\sum_{a \in A \backslash\{b\}} u_{a}^{*} \delta_{a k}^{w}\right) \\
& =\sum_{a \in A} t_{a}\left(\mathbf{v}^{\max }\right)+\lambda_{w k_{0}}\left(\mathbf{u}^{*}\right)-\left(M+\sum_{a \in \bar{A} \backslash b\}} u_{a}^{*} \delta_{a k}^{w}\right) \\
& \leq \sum_{a \in A} t_{a}\left(\mathbf{v}^{\max }\right)-\left(1-\gamma_{1}\right) M
\end{aligned}
$$

According to eqns. (61), (63) and (64), we can obtain 


$$
v_{w b}\left(\mathbf{u}^{*}\right) \leq \bar{q}_{w} \sum_{k \in R_{w b}} \operatorname{Pr}\left(\zeta_{w k}-\zeta_{w k_{0}} \leq \sum_{a \in A} t_{a}\left(\mathbf{v}^{\max }\right)-\left(1-\gamma_{1}\right) M\right)
$$

According to eqn. (53), eqn. (65) implies that

$$
v_{w b}\left(\mathbf{u}^{*}\right) \leq \frac{\gamma_{2} \times \min _{a \in A}\left\{H_{a}\right\}}{|W|}<\frac{H_{b}}{|W|}
$$

Case 2: Assume $\lambda_{w k}\left(\mathbf{u}^{*}\right)$ for each path $k \in R_{w}$ is larger than $\gamma_{1} M$, i.e.,

$$
\lambda_{w k}\left(\mathbf{u}^{*}\right)=\sum_{a \in \bar{A}} u_{a}^{*} \delta_{a k}^{w}>\gamma_{1} M, \forall k \in R_{w}
$$

Therefore, the satisfaction $S_{w}\left(\mathbf{c}_{w}\left(\mathbf{f}\left(\mathbf{u}^{*}\right)\right)+\lambda_{w}\left(\mathbf{u}^{*}\right)\right)$ fulfills the condition:

$$
S_{w}\left(\mathbf{c}_{w}\left(\mathbf{f}\left(\mathbf{u}^{*}\right)\right)+\boldsymbol{\lambda}_{w}\left(\mathbf{u}^{*}\right)\right) \geq \gamma_{1} M
$$

According to the assumption that the OD demand function is non-increasing and eqn. (52), we have

$$
D_{w}\left(S_{w}\left(\mathbf{c}_{w}\left(\mathbf{f}\left(\mathbf{u}^{*}\right)\right)+\lambda_{w}\left(\mathbf{u}^{*}\right)\right)\right) \leq D_{w}\left(\gamma_{1} M\right) \leq \frac{\gamma_{2}}{|W|} \min _{a \in \bar{A}}\left\{H_{a}\right\}<\frac{H_{b}}{|W|}
$$

In other words,

$$
v_{w b}\left(\mathbf{u}^{*}\right)=\sum_{k \in R_{w}} f_{w k}\left(\mathbf{u}^{*}\right) \delta_{b k}^{w} \leq D_{w}\left(S_{w}\left(\mathbf{c}_{w}\left(\mathbf{f}\left(\mathbf{u}^{*}\right)\right)+\lambda_{w}\left(\mathbf{u}^{*}\right)\right)\right)<\frac{H_{b}}{|W|}
$$

Based on eqns. (66) and (70), we can conclude that

$$
v_{b}\left(\mathbf{u}^{*}\right)=\sum_{w \in W} v_{w b}\left(\mathbf{u}^{*}\right)<H_{b}
$$

However, eqn. (71) contradicts eqn. (57), thus the hypothesis $u_{b}^{*}=M$ is incorrect. In other words,

$$
u_{a}^{*}<M, \forall a \in \bar{A}
$$

To further prove the necessary conditions, we construct the following two specific vectors for any link $a \in \bar{A}$ :

$$
\begin{gathered}
\mathbf{u}^{\prime}(a)=\left(u_{b}^{\prime}=u_{b}^{*}, b \in A \backslash\{a\}, u_{a}^{\prime}=\left(1-\gamma_{1}\right) u_{a}^{*}+\gamma_{1} M, a \in \bar{A}\right)^{\mathrm{T}} \\
\mathbf{u}^{\prime \prime}(a)=\left(u_{b}^{\prime \prime}=u_{b}^{*}, b \in A \backslash\{a\}, u_{b}^{\prime \prime}=\gamma_{1} u_{a}^{*}, a \in \bar{A}\right)^{\mathrm{T}}
\end{gathered}
$$

Since these two vectors are in set $\Omega$, substituting vector $\mathbf{u}$ of VI model $V I(\boldsymbol{\Phi}, \Omega)$ with these two particular vectors, respectively, yields that

$$
\begin{gathered}
{\left[H_{a}-v_{a}^{*}\left(\mathbf{u}^{*}\right)\right] \times\left[\gamma_{1}\left(M-u_{a}^{*}\right)\right] \geq 0} \\
{\left[H_{a}-v_{a}^{*}\left(\mathbf{u}^{*}\right)\right] \times\left[\left(\gamma_{1}-1\right) u_{a}^{*}\right] \geq 0}
\end{gathered}
$$

Since $M-u_{a}^{*}>0$, eqn. (75) implies that

$$
v_{a}^{*}\left(\mathbf{u}^{*}\right) \leq H_{a}, \forall a \in \bar{A}
$$

In accordance with the fact that $u_{a}^{*} \geq 0$, we thus have

$$
\left(H_{a}-v_{a}^{*}\left(\mathbf{u}^{*}\right)\right) u_{a}^{*} \geq 0, \forall a \in \bar{A}
$$


Hence, eqn. (76) in conjunction with eqn. (78) imply that

$$
\left(H_{a}-v_{a}^{*}\left(\mathbf{u}^{*}\right)\right) u_{a}^{*}=0, \forall a \in \bar{A}
$$

In other words, $\mathbf{u}^{*}$ and $\mathbf{v}\left(\mathbf{u}^{*}\right)$ fulfill the generalized SUE conditions.

Sufficient condition

Suppose that $\mathbf{u}^{*}$ and $\mathbf{v}\left(\mathbf{u}^{*}\right)$ satisfy the generalized SUE conditions expressed by eqns. (9) -(12). And using the similar tactic to eqns. (56) to (72), we can show that $0 \leq u_{a}^{*}<M, \forall a \in \bar{A}$. In addition, eqns. (9) to (12) imply that

$$
\begin{gathered}
\mathbf{H}-\mathbf{v}_{\bar{A}}\left(\mathbf{u}^{*}\right) \geq \mathbf{0} \\
\left(\mathbf{H}-\mathbf{v}_{\bar{A}}\left(\mathbf{u}^{*}\right)\right)^{\mathrm{T}} \mathbf{u}^{*}=0
\end{gathered}
$$

For any $\mathbf{u} \in \Omega$, we have $\mathbf{u} \geq 0$. Thus, according to eqns. (80)-(81), it follows that

$$
\left(\mathbf{H}-\mathbf{v}_{\bar{A}}\left(\mathbf{u}^{*}\right)\right)^{\mathrm{T}} \mathbf{u} \geq\left(\mathbf{H}-\mathbf{v}_{\bar{A}}\left(\mathbf{u}^{*}\right)\right)^{\mathrm{T}} \mathbf{u}^{*}
$$

Rearranging eqn. (82) yields that

$$
\left(\mathbf{H}-\mathbf{v}_{\bar{A}}\left(\mathbf{u}^{*}\right)\right)^{\mathrm{T}}\left(\mathbf{u}-\mathbf{u}^{*}\right) \geq 0
$$

That is,

$$
\boldsymbol{\Phi}\left(\mathbf{u}^{*}\right)^{\mathrm{T}}\left(\mathbf{u}-\mathbf{u}^{*}\right) \geq 0
$$

This then proved the sufficient condition.

Since $V I(\boldsymbol{\Phi}, \Omega)$ has at least one solution, Proposition 4 therefore confirms the existence property of solution for the generalized SUE conditions. Moreover, since the first VI model $V I\left(\boldsymbol{\Phi}, \mathfrak{R}_{+}^{|\bar{A}|}\right)$ is equivalent to the generalized SUE conditions, we thus know that $\operatorname{VI}\left(\boldsymbol{\Phi}, \mathfrak{R}_{+}^{|\bar{A}|}\right)$ also has at least one solution, with the aid of $\operatorname{VI}(\boldsymbol{\Phi}, \Omega)$. As vector function $\boldsymbol{\Phi}(\mathbf{u})$ is only monotone rather than strictly or strongly monotone, solution to the restricted VI model $V I(\boldsymbol{\Phi}, \Omega)$ may not be unique. In other words, the optimal Lagrangian multipliers involved in generalized SUE conditions are not unique. While, it should be pointed out the SUE link flow solution is unique for any given optimal Lagrangian multiplier solutions.

The three important properties of vector function $\boldsymbol{\Phi}(\mathbf{u})$ are all available for $\operatorname{VI}(\boldsymbol{\Phi}, \Omega)$. Its monotone and uniform Lipschitz-continuous properties are prerequisites for the global convergence of many solution algorithms. To sum up, the new VI model $V I(\boldsymbol{\Phi}, \Omega)$ not only helps us in demonstrating the existence of solution to asymmetric SUE problem with elastic demand and link capacity constraint, but also inherits the three important properties proven in Section 3.1. These properties can guarantee the global convergence of some algorithms for solving the monotone VI models. 


\section{SOLUTION ALGORITHM}

As vector function $\boldsymbol{\Phi}(\mathbf{u})$ of VI model $V I(\Phi, \Omega)$ is an implicit function, it is thus quite burdensome to evaluate its gradient. On the other hand, with any given Lagrangian-multiplier vector $\mathbf{u}$, the value of vector function $\boldsymbol{\Phi}(\mathbf{u})$ is link flow solution of an asymmetric SUE problem with elastic demand but no link capacity constraint. Thus, it can be calculated efficiently by the convergent CA method proposed by Cantarella (1997). In addition, the projection operation on set $\Omega$ of VI model $\operatorname{VI}(\boldsymbol{\Phi}, \Omega)$ is effortless. Propositions 1 and 3 imply that the restricted VI model is monotone and uniform Lipschitz-continuous. All these facts inspire us to choose a projection type method for solving the restricted VI model $V I(\Phi, \Omega)$ because it only calculates value of the vector function and implants the projection operations at each iteration (Wu, 1991; Chen et al., 2001; Facchinei and Pang, 2003).

The prediction-correction (PC) algorithm proposed by Korpelevich (1976) is a projection type method with the global convergence when solving a monotone and Lipschitz-continuous VI model. For solving $V I(\boldsymbol{\Phi}, \Omega)$, the PC algorithm at iteration $n$ consists of the prediction and correction steps as follows:

Prediction:

$$
\overline{\mathbf{u}}^{(n+1)}=P_{\Omega}\left[\mathbf{u}^{(n)}-\eta^{(n)} \boldsymbol{\Phi}\left(\mathbf{u}^{(n)}\right)\right]
$$

Correction:

$$
\mathbf{u}^{(n+1)}=P_{\Omega}\left[\mathbf{u}^{(n)}-\eta^{(n)} \boldsymbol{\Phi}\left(\overline{\mathbf{u}}^{(n+1)}\right)\right]
$$

where step size $\eta^{(n)}>0$ should satisfy the following condition with a predetermined parameter $v \in(0,1)$ :

$$
\eta^{(n)} \leq v \frac{\left\|\mathbf{u}^{(n)}-\overline{\mathbf{u}}^{(n+1)}\right\|_{2}}{\left\|\boldsymbol{\Phi}\left(\mathbf{u}^{(n)}\right)-\boldsymbol{\Phi}\left(\overline{\mathbf{u}}^{(n+1)}\right)\right\|_{2}}
$$

Existence of this step size is assured from the uniform Lipschitz-continuity of vector function

$\boldsymbol{\Phi}(\mathbf{u})$ and it can be determined by the Armijo rule starting with $\tilde{\eta}=\eta^{(n-1)}$ within finite iterations (Bertsekas, 1976):

$$
\tilde{\eta}=\frac{2}{3} \min \left\{\tilde{\eta}, \frac{\left\|\mathbf{u}^{(n)}-\overline{\mathbf{u}}^{(n+1)}\right\|_{2}}{\left\|\boldsymbol{\Phi}\left(\mathbf{u}^{(n)}\right)-\boldsymbol{\Phi}\left(\overline{\mathbf{u}}^{(n+1)}\right)\right\|_{2}}\right\}
$$

The Armijo rule shown in eqn. (88) makes step size $\eta^{(n)}$ monotonically decreasing with the increase of iterations and it makes convergent speed of PC algorithm slow down. In addition, the prediction and correction steps both take the same step size $\eta^{(n)}$. He and Liao 
(2002) improved the PC algorithm by proposing a self-adaptive step size adjustment scheme in the correction step and named it as self-adaptive PC algorithm. The self-adaptive PC algorithm also slightly enlarges the step size at each iteration if possible and has linear convergent rate (He and Liao, 2002). The self-adaptive PC algorithm incorporating the CA method for solving $V I(\boldsymbol{\Phi}, \Omega)$ is given below.

\section{Self-adaptive PC incorporating CA method}

Step 0: (Initialization) Choose an initial vector $\mathbf{u}^{(1)}=\left(u_{a}^{(1)}=0, a \in \bar{A}\right)^{\mathrm{T}}$, three constants $0<\kappa_{2}<\kappa_{1}<1, \gamma \in(0,2)$, and initial step size $\eta^{(1)}>0$. Let the number of iterations $n=1$.

Step 1: (Projection with step size adjustment) Find vector $\overline{\mathbf{u}}^{(n)}$ with a proper step size $\eta^{(n)}$ through the following procedure:

Step 1.1: For vector $\mathbf{u}^{(n)}$, first calculate the parametric SUE link flow vector $\mathbf{v}\left(\mathbf{u}^{(n)}\right)=\left(v_{a}\left(\mathbf{u}^{(n)}\right), a \in A\right)^{\mathrm{T}}$ using the CA method and then compute $\boldsymbol{\Phi}\left(\mathbf{u}^{(n)}\right)=\left(H_{a}-v_{a}\left(\mathbf{u}^{(n)}\right), a \in \bar{A}\right)^{\mathrm{T}}$.

Step 1.2: Find vector $\overline{\mathbf{u}}^{(n)}$ by the projection:

$$
\overline{\mathbf{u}}^{(n)}=P_{\Omega}\left[\mathbf{u}^{(n)}-\eta^{(n)} \mathbf{\Phi}\left(\mathbf{u}^{(n)}\right)\right]
$$

Step 1.3: For the vector $\overline{\mathbf{u}}^{(n)}$ obtained in Step 1.2, calculate parametric SUE link flow solution $\mathbf{v}\left(\overline{\mathbf{u}}^{(n)}\right)=\left(v_{a}\left(\overline{\mathbf{u}}^{(n)}\right), a \in A\right)^{\mathrm{T}}$ using the CA method and then calculate $\boldsymbol{\Phi}\left(\overline{\mathbf{u}}^{(n)}\right)=\left(H_{a}-v_{a}\left(\overline{\mathbf{u}}^{(n)}\right), a \in \bar{A}\right)^{\mathrm{T}}$.

Step 1.4: Calculate ratio $r^{(n)}$ :

$$
r^{(n)}=\eta^{(n)}\left\|\boldsymbol{\Phi}\left(\mathbf{u}^{(n)}\right)-\boldsymbol{\Phi}\left(\overline{\mathbf{u}}^{(n)}\right)\right\|_{2} /\left\|\mathbf{u}^{(n)}-\overline{\mathbf{u}}^{(n)}\right\|_{2}
$$

If $r^{(n)} \leq \kappa_{1}$, go to Step 2; otherwise go to Step 1.5

Step 1.5: Reduce the step size according to

$$
\eta^{(n)}=\frac{2}{3} \eta^{(n)} \min \left\{1, \frac{1}{r^{(n)}}\right\}
$$

and go to Step 1.1

Step 2: (Stop check) If $\left\|\mathbf{u}^{(n)}-\overline{\mathbf{u}}^{(n)}\right\| \leq \varepsilon_{2}$, where $\varepsilon_{2}$ is a predetermined positive tolerance, then stop; otherwise, go to Step 3.

Step 3: (Correction with self-adaptive step size adjustment) Based on $\mathbf{u}^{(n)}, \overline{\mathbf{u}}^{(n)}$ and $\eta^{(n)}$, calculate a proper step size $\alpha^{(n)}$ for correction and then get an updated vector $\mathbf{u}^{(n+1)}$ : Step 3.1: Calculate another step size $\alpha^{(n)}$ as per the formula: 


$$
\alpha^{(n)}=\gamma \times \eta^{(n)} \times \sum_{a \in \bar{A}}\left[\left(u_{a}^{(n)}-\bar{u}_{a}^{(n)}\right) \times h_{a}^{(n)}\right] / \sum_{a \in \bar{A}}\left(h_{a}^{(n)}\right)^{2}
$$

where

$$
h_{a}^{(n)}=\left(u_{a}^{(n)}-\bar{u}_{a}^{(n)}\right)+\eta^{(n)}\left(\Phi_{a}\left(\mathbf{u}^{(n)}\right)-\Phi_{a}\left(\overline{\mathbf{u}}^{(n)}\right)\right), a \in \bar{A}
$$

Step 3.2: Update the vector $\mathbf{u}^{(n+1)}$ by this projection:

$$
\mathbf{u}^{(n+1)}=P_{\Omega}\left[\mathbf{u}^{(n)}-\alpha^{(n)} \boldsymbol{\Phi}\left(\overline{\mathbf{u}}^{(n)}\right)\right]
$$

Step 3.3: Enlarge step size $\eta^{(n)}$ according to the following scheme:

$$
\eta^{(n+1)}=\frac{3}{2} \eta^{(n)} \text { if } \eta^{(n)}\left\|\boldsymbol{\Phi}\left(\mathbf{u}^{(n)}\right)-\boldsymbol{\Phi}\left(\overline{\mathbf{u}}^{(n)}\right)\right\|_{2} /\left\|\mathbf{u}^{(n)}-\overline{\mathbf{u}}^{(n)}\right\|_{2} \leq \kappa_{2}
$$

$$
\eta^{(n+1)}=\eta^{(n)} \text {, otherwise. Let } n=n+1 \text { and go to Step } 1 \text {. }
$$

The uniform Lipschitz-continuity of function vector $\boldsymbol{\Phi}(\mathbf{u})$ ensures that condition $r^{(n)} \leq \kappa_{1}$ in Step 1.4 can be fulfilled in the finite iterations, and moreover inf $\left\{\eta^{(n)}\right\}=\eta_{\text {min }}>0$ (He and Liao, 2002). The stop criterion utilized in Step 2 comes from the fact that $\mathbf{u}^{*}$ is a solution of VI model $V I(\Phi, \Omega)$ if and only if (Nagurney, 1993):

$$
\mathbf{u}^{*}=P_{\Omega}\left[\mathbf{u}^{*}-\rho \Phi\left(\mathbf{u}^{*}\right)\right]
$$

where $\rho$ is an arbitrary positive parameter. In reality,

$$
\left\|\mathbf{u}^{(n)}-\overline{\mathbf{u}}^{(n)}\right\|_{2}=\left\|\mathbf{u}^{(n)}-P_{\Omega}\left[\mathbf{u}^{(n)}-\eta^{(n)} \boldsymbol{\Phi}\left(\mathbf{u}^{*}\right)\right]\right\|_{2}
$$

is an error bound value of $\mathbf{u}^{(n)}$ and it would be equal to zero only if $\mathbf{u}^{(n)}$ is a solution of VI model $V I(\boldsymbol{\Phi}, \Omega)$. We highlight that global convergence of the self-adaptive PC algorithm incorporating CA method is assured because the restricted VI model $V I(\boldsymbol{\Phi}, \Omega)$ is monotone and uniform Lipschitz-continuous. The self-adaptive PC algorithm is proven to have a linear convergent speed, thus it is more efficient than some other methods with non-adaptive step sizes and sub-linear convergent speed, e.g., the Method of Successive Average.

\section{NUMERICAL EXAMPLE}

This section uses the well-known Sioux-Falls network, shown in Figure 1, to numerically assess performance of the proposed VI model and self-adaptive PC algorithm for solving the asymmetric SUE problem with elastic demand and link capacity constraints.

(Figure 1 should be inserted around here)

The Sioux-Falls network consists of 24 nodes, 76 links and 528 OD pairs. Travel demand between each OD pair $w \in W$ for this example is assumed to be a function of the satisfaction:

$$
q_{w}=\bar{q}_{w} \times \exp \left(-\rho \times S_{w}\left(\mathbf{c}_{w}(\mathbf{f})\right)\right), w \in W
$$


where the positive parameter $\rho$ is taken as 0.01 , and the upper bound of OD demand, $\bar{q}_{w}$, can be found in the website of transportation network test problems (Bar-Gera, 2012), which are omitted here due to the space limit. This example adopts the following link travel time functions:

$$
t_{a}(\mathbf{v})=t_{a}^{0}\left(1+0.03 \times\left(\frac{v_{a}+0.5 v_{\hat{a}}}{1.5 h_{a}}\right)^{4}\right), a \in A
$$

where $\hat{a}$ is the opposite link of $a$ on a two-way street, and $t_{a}^{0}$ is the free-flow travel time of link $a$ and $h_{a}$ is the traffic flow capacity of link $a \in A$. As these free-flow travel times and traffic flow capacities can be found in Bar-Gera (2012), they are not listed here. It is not difficult to check that Jacobian matrix of all these link travel time functions is asymmetric. It is further assumed that there is a threshold levied on each link in set $\bar{A}=\{3,10,15,30,50,70\}$ that three scenarios of the thresholds of these six links will be investigated, namely;

$$
v_{a} \leq H_{a}^{s}, a \in \bar{A}, s=1,2,3
$$

where threshold $H_{a}^{s}$ of link $a$ for Scenario $s(s=1,2,3)$ as well as its traffic flow capacity $h_{a}$ are given in Table 1.

(Table 1 should be inserted around here)

The behavior of network users in route choice for this example is assumed to follow the probit-based SUE principle due to its good representativeness. A link-based representation is adopted for the probit-based SUE problem (see, Chapter 11.2, Sheffi, 1985). Namely, travel time of each link perceived by network users is assumed to be normally distributed and statistically independent:

$$
\begin{gathered}
T_{a}(\mathbf{v})=t_{a}(\mathbf{v})+\xi_{a}, a \in A \\
\xi_{a}: N\left(0, \beta t_{a}^{0}\right), a \in A
\end{gathered}
$$

where $\xi_{a}$ is the perception error on travel time of link $a$, $t_{a}^{0}$ is the free-flow travel time and $\beta$ is a predetermined proportion parameter, which is set to be 0.1 for this example.

It should be pointed out that in each iteration of the CA method, a probit-based stochastic network loading problem should be solved. For the probit-based SUE problem with elastic demand and asymmetric link travel time functions, its stochastic network loading problem is solved by a two-stage Monte Carlo simulation based method proposed by Meng and Liu (2012). It should be noted that the value of $S_{w}\left(\mathbf{c}_{w}(\mathbf{f})\right)$ can be obtained during the Monte Carlo simulation to calculate the travel demand in eqn. (98). Based on the sampled travel 
times, although in some extreme case, the value of $\min _{k \in R_{w}}\left\{C_{w k}(\mathbf{f})\right\}$ might be negative, it is unlikely that its mean value $S_{w}\left(\mathbf{c}_{w}(\mathbf{f})\right)$ can become a negative value. Thus, it guarantees that $q_{w} \leq \bar{q}_{w}$

Parameters in the self-adaptive PC algorithms are set as follows: $\kappa_{1}=0.9, \kappa_{2}=0.1$, $\gamma=1.8, \eta^{(0)}=1.0$ and $\varepsilon_{2}=0.01$. In order to reflect the convergence trend of the self-adaptive PC algorithm incorporating CA method more precisely and apparently, we take the following logarithmic value of the error bound shown in eqn. (97) as a performance index:

Logarithmic value of the error bound $=\log _{10}\left(\left\|\mathbf{u}^{(n)}-\overline{\mathbf{u}}^{(n)}\right\|_{2}\right)$

Before calculating the addressed problem, the SUE problem with no capacity constraint is first solved on this network example. The equilibrium link flows on each link in $\bar{A}=\{3,10,15,30,50,70\}$ is provided in the last column of Table 1, entitled "SUE Flows". It shows that the SUE flows on nearly all the links (except link 3) are larger than the capacity constraints in all the three scenarios. In particular, the SUE flows on links 10, 15, 30, 70 are even much higher than the physical capacity. Therefore, it clearly validates the significance of capacity constraints, since the SUE problem with no capacity constraint would give unrealistic equilibrium flows.

The self-adaptive PC algorithm is then used to solve the capacity constrained SUE problem in terms of the three scenario provided in Table 1. Figure 2 depicts the logarithmic value of the error bound versus the CPU times used for solving each scenario. It clearly shows that the algorithm can monotonically converge to a solution of VI model $\operatorname{VI}(\boldsymbol{\Phi}, \Omega)$.

(Figure 2 should be inserted around here)

Table 2 gives the resultant Lagrangian multiplier vector $\mathbf{u}^{*}$ and corresponding flowthreshold ratio, $v_{a}\left(\mathbf{u}^{*}\right) / H_{a}^{s}, a \in \bar{A}$, for each scenario $s=1,2,3$. Table 2 indicates that the flow-threshold ratio does not exceed 1.0 (on some links it is 1.01 caused by the computation error) and the generalized Lagrangian multiplier takes positive value only on the links with a flow-threshold ratio close to 1.0. These two phenomena tally with eqns. (10)-(12), which numerically verify the effectiveness of VI model $V I(\Phi, \Omega)$ and self-adaptive PC algorithm. In addition, it can be seen that as the values of threshold are getting larger from Scenario 1 to Scenario 3, the relevant optimal Lagrangian multipliers on most links become larger. This implies that it would be more costly to restrict the link flow to a much lower level.

(Table 2 should be inserted around here) 
To further see the impacts of demand level on the optimal Lagrangian multipliers, a sensitivity test is carried out on the parameter $\rho$ in the demand function (98). Without loss of generality, the first scenario of threshold setting is taken for this test, and then the value of $\rho$ is changed from 0.05 to 0.01 and 0.005 . The optimal Lagrangian multipliers obtained by the self-adaptive PC algorithm in these three cases are provided in Table 3. It should be noted that a smaller value of $\rho$ implies that the demand level is higher and the network congestion level is higher accordingly. The data in Table 3 shows that the generalized SUE conditions can still be fulfilled, and as the demand level ascends, the values of optimal Lagrangian multipliers also increases, meaning that it is more difficult to maintain the threshold constraints at a higher demand level.

(Table 3 should be inserted around here)

Another complementary test is carried out for the uniqueness of the optimal Lagrangian multiplier. Here, the first scenario of threshold is adopted and the value of $\rho$ in the demand function is taken as 0.05 . We performed four cases of tests, where the initial values of Lagrangian multipliers are set as 0.0, 1.0, 2.0, 3.0 and 4.0, respectively. The numerical results show that the optimal Lagrangian multipliers for these four cases are identical to the values shown in the first column of Table 3. Hence, although the uniqueness of the optimal Lagrangian multiplier cannot be theoretically proven, the optimum of this network example with strictly monotone demand function is probably unique.

The data in Tables 2 and 3 indicate that the flows on the constrained links have been restricted to be less than or equal to the predetermined threshold, via adding an extra terms on the link travel costs, which are equal to the optimal Lagrangian multipliers. In practice, this extra term on the constrained links can be realized by setting a signal light or toll charge (Yang and Bell, 1997). Therefore, when the network authority has decided to restrict the flow on any link (usually in the urban area or highways) with a threshold, the methodology proposed in this paper can be adopted to calculate the signal setting and proper toll charge on such links.

Furthermore, from the viewpoint of the network authority, setting the threshold values on constrained links is also a big concern which may largely affect the network efficiency. Hence, in practice, it is necessary to further investigate the design of these threshold capacities, with the aim of optimizing the efficiency/benefit in the entire road network. Such kind of study can be formulated as a bi-level model, taking the model proposed in this paper 
as the lower level. Also, the solution algorithm in Section 4 can be used to evaluate each feasible pattern of threshold capacities.

\section{CONCLUSIONS}

This paper dealt with the asymmetric SUE problem with elastic demand and link capacity constraints. After defining the generalized SUE conditions in terms of the generalized link travel times, we developed a VI model for these generalized SUE conditions by using the parametric SUE link flow solution. It was rigorously proven that the VI model is equivalent to the generalized SUE conditions. More importantly, this VI model is monotone and uniform Lipschitz-continuous. The self-adaptive PC algorithm incorporating CA method was then employed for solving the VI model and its global convergence is assured. It can be concluded that this study has completely solved the open question, namely, to provide an equivalent model and global convergent algorithm for solving the general SUE problem with elastic demand, asymmetric link travel time functions and link capacity constraints.

The optimal Lagrangian multipliers may not be unique for a particular road network, yet the solution algorithm in Section 4 can only converge to one optimal solution. Hence, an interesting future research topic is to search for the other optimal solutions based on the one obtained by self-adaptive PC algorithm. Likewise to the work of Hearn and Ramana (1998) for first-best congestion pricing, the other patterns of Lagrangian multipliers that can generate the same equilibrium flows could be achieved.

It can be seen from the numerical test that a long execution time is required, which is mainly caused by the large computation burden of Monte Carlo simulation based solution method for the probit-based SUE problem. Once the values of Lagrangian multipliers change in one iteration of the PC algorithm, it would take thousands of simulations to get the corresponding equilibrium flows, where each simulation is an all-or-nothing assignment based on sampled travel times. This paper mainly focuses on the theoretical analysis, yet when applied in practice, the long computation time would restrict the implementations of proposed methodology. A good way to reduce the computation time for practical implementations is to calculate the Monte Carlo simulation on a parallel computing system. The study by Liu and Meng (2011) shows that the computational speed of this Monte Carlo simulation can be accelerated for over 60 times when 100 processors are harnessed for the calculation. In future, it is necessary to test the performance of the distributed computing approaches for solving the capacity constrained SUE problem. 
Future efforts are also needed to further extend the proposed methodology to the cases of multi-user classes, multi-vehicle types, as well as dynamic traffic assignment.

\section{REFERENCES}

Abdulaal, M. and Leblanc, L.J. 1979. Methods for combining modal split and equilibrium assignment models. Transportation Science, 13(4), 292-314.

Baillon, J.B., and Cominetti, R., 2008. Markovian traffic equilibrium. Mathematical Programming, 111, 33-56.

Bar-Gera, H., 2012, Transportation Network Test Problems Website. Available from: http://www.bgu.ac.il/ bargera/tntp/. [Accessed at Sep 25 ${ }^{\text {th }}, 2012$ ]

Bekhor, S. and Toledo, T., 2005. Investigating path-based solution algorithms to the stochastic user equilibrium problem. Transportation Research Part B, 39(3), 279-295.

Bekhor, S., Toledo, T., and Prashker, J.N., 2008. Effects of choice set size and route choice models on path-based traffic assignment. Transportmetrica, 4(2), 117-133.

Bell, M.G.H., 1995. Stochastic user equilibrium assignment in networks with queues. Transportation Research Part B, 29, 125-137.

Bell, M.G.H. and Iida, Y., 1997. Transportation Network Analysis. John Wiley \& Sons.

Bertsekas, D.P., 1976. On the Goldstein-Levitin-Polyak gradient projection method. IEEE transactions on automatic control, Vol. AC-21, No. (2), 174-184

Cantarella, G.E., 1997. A general fixed-point approach to multimode multi-user equilibrium assignment with elastic demand, Transportation Science, 31, 107-128.

Cantarella, G.E., and Cascetta, E., 2009. Advanced models for traffic assignment to transportation networks. Chapter 6 in Cascetta, E. (ed.) Transportation Systems Analysis. Springer.

Chen, M., Alfa, A.S., 1991. Algorithms for solving Fisk's stochastic traffic assignment model. Transportation Research Part B, 25, 405 - 412.

Chen, A., Lo, H.K., Yang, H., 2001. A self-adaptive projection and contraction algorithm for the traffic assignment problem with path-specific costs. European Journal of Operational Research, 135, 27-41

Chen, A., Xu, X., Ryu, S., and Zhou, Z., 2012. A self-adaptive Armijo stepsize strategy with application to traffic assignment models and algorithms. Transportmetrica, doi:10.1080/18128602.2011.653999.

Connors, R.D., and Sumalee, A., 2009. A network equilibrium model with travelers' perception of stochastic travel times. Transportation Research Part B, 43, 614-624.

Dafermos, S.C., 1980. Traffic equilibrium and variational inequalities. Transportation Science, 14, 42-54.

Dafermos, S.C., 1982. Relaxation algorithms for the general asymmetric traffic equilibrium problem. Transportation Science, 16, 231-240.

Dafermos, S.C., 1983. An iterative scheme for variational equalities. Mathematical Programming, 26, 40-47.

Daganzo, C.F., 1982. Unconstrained extremal formulation of some transportation equilibrium problems. Transportation Science, 16, 332-360.

Daganzo, C.F., 1983. Stochastic network equilibrium with multiple vehicle types and asymmetric, indefinite link cost Jacobians, Transportation Science, 17, 282-300.

Daganzo, C.F. and Sheffi, Y., 1977. On stochastic models of traffic assignment. Transportation Science, 11(3), 253-274.

Damberg, O., Lundgren, J.T., Patriksson, M., 1996. An algorithm for the stochastic user equilibrium problem. Transportation Research Part B, 30, 115 - 131. 
Facchinei, F. and Pang, J.S., 2003. Finite-dimensional Variational Inequalities and Complementarity Problems: Volumes 1 \&2. Springer-Verlag.

Ferrari, P., 1997. Capacity constraint in urban transport networks. Transportation Research Part B, 31, 291-301.

Florian, M. and Spiess, H., 1982. The convergence of diagonalization algorithms for asymmetric network equilibrium problems. Transportation Research Part B, 16, 477483.

Friesz, T.L., Harker, P.T., and Tobin, R.L., 1984. Alternative algorithm for the general network spatial price equilibrium problem. Journal of Regional Science, 24(4), 475507.

He, B.S. and Liao L.Z., 2002. Improvements of some projection methods for monotone nonlinear variational inequalities. Journal of Optimization Theory and Applications, 112(1), 111-128

Hearn, D.W. and Lawphongpanich, S., 1990. A dual ascent algorithm for traffic assignment problems. Transportation Research Part B, 24, 423-430.

Hearn, D.W. and Ramana M.V., 1998. Solving congestion toll pricing models. In: Marcotte, P., Nguyen, S., (eds.) Equilibrium and advanced transportation modelling. Kluwer Academic Publishers, Norwell, MA, pp. 109 - 124.

Hearn, D.W. and Ribera, J., 1980. Bounded flow equilibrium problems by penalty methods. Proceedings of IEEE International Conference on Circuits and Computers 1, IEEE, New York, 162-166.

Huang, H.-J. and Li, Z., 2007. A multiclass, multicriteria logit-based traffic equilibrium assignment model under ATIS. European Journal of Operational Research, 176(3), 1464-1477.

Inouye, H., 1987. Traffic equilibria and its solution in congested road networks. In: Genser, R. (Ed.), Proceedings of IFAC Conference on Control in Transportations, 267-272.

Korpelevich, G.M., 1976. The extragradient method for finding saddle points and other problems, Ekonomika Matematicheskie Metody 12, 747-756.

Larsson, T. and Patriksson, M., 1995. An augmented Lagrangean dual algorithm for link capacity side constrained traffic assignment problems. Transportation Research Part B, 29(6), 433-455.

Larsson, T. and Patriksson, M., 1999. Side constrained traffic equilibrium models - analysis, computation and applications. Transportation Research Part B, 33(4), 233-264.

Liu, Z., Meng, Q., 2011. Distributed computing approaches for large-scale probit-based Stochastic User Equilibrium problems. Journal of Advanced Transportation, DOI: 10.1002/atr.177.

Maher, M., 1998. Algorithms for logit-based stochastic user equilibrium assignment. Transportation Research Part B, 32 (8), 539 - 549.

Maher M.J. and Hughes P.C., 1997. A probit-based stochastic user equilibrium assignment model. Transportation Research Part B, 31(4), 341-355.

Maher, M.J. and Zhang X., 2000. Formulation and algorithms for the problem of stochastic user equilibrium assignment with elastic demand. 8th EURO Working Group meeting on Transportation, Rome, September 2000.

Meng, Q., Lam, W.H.K., and Yang, L., 2008. General stochastic user equilibrium traffic assignment problem with link capacity constraints. Journal of Advanced Transportation, 42, 429-465.

Meng, Q., and Liu, Z., 2012. Mathematical models and computational algorithms for probitbased asymmetric stochastic user equilibrium problem with elastic demand. Transportmetrica, 8(4), 261-290. 
Mirchandani, P., and Soroush, H., 1987. Generalized traffic equilibrium with probabilistic travel times and perceptions. Transportation Science, 21(3), 133-152.

Nagurney, A., 1993. Network economics: a variational inequality approach. Kluwer Academic publishers, Dordrecht, Boston, London.

Nie, Y., Zhang, H.M. and Lee, D.H., 2004. Models and algorithms for the traffic assignment problem with link capacity constraints. Transportation Research Part B, 38(4), 285312.

Ortega, J.M. and Rheinboldt, W.C., 1970. Iterative Solution of Nonlinear Equations in Several Variables. Academic Press, New York and London.

Patriksson, M., 1994a. The Traffic Assignment Problems: Models and Methods. VSP, Utrecht, the Netherlands.

Patriksson, M., 1994b. On the convergence of descent methods for monotone variational inequalities. Operations Research Letters, 16 (5), 265-269.

Patriksson, M., 2008. On the applicability and solution of bilevel optimization models in transportation science: a study on the existence, stability and computation of optimal solutions to stochastic mathematical programs with equilibrium constraints. Transportation Research Part B, 42, 843-860.

Pravinvongvuth, S., Chen, A., 2005. Adaptation of the paired combinatorial logit model to the route choice problem, Transportmetrica, 1(3), 223-240.

Sheffi, Y. 1985. Urban Transportation Networks: Equilibrium analysis with Mathematical Programming Models. Prentice-Hall, INC, Englewood Cliffs, New Jersey.

Sheffi, Y. and Powell, W.B., 1982. An algorithm for the equilibrium assignment problem with random link times. Networks, 12(2), 191-207.

Smith, M.J., 1979. The existence, uniqueness and stability of traffic equilibrium. Transportation research Part B, 13, 295-304.

Wardrop, J.G., 1952. Some Theoretical Aspects of Road Traffic Research. Proceedings, Institution of Civil Engineers II(1), 325-378.

Watling, D., 1998. Perturbation stability of the asymmetric stochastic equilibrium assignment model. Transportation Research Part B, 32, 155-171.

Watling, D., 2006. User equilibrium traffic network assignment with stochastic travel times and late arrival penalty. European Journal of Operational Research, 175, 1539-1556.

Wei, C., Asakura, Y., Iryo, T., 2012. The posterior probability distribution of traffic flow: A new scheme for the assignment of stochastic traffic flow. Transportmetrica, DOI: 10.1080/18128602.2012.661799.

Wu, J.H., 1991. A Study of Monotone Variational Inequalities and Their Application to Network Equilibrium Problems. Ph.D Dissertation, Centre for Research on Transportation, University of Montreal, Canada.

Yang, H. and Bell, M.G.H., 1997. Traffic restraint, road pricing and network equilibrium. Transportation Research Part B, 31(4), 303-314.

Yang, H. and Huang, H.J., 2005. Mathematical and Economic Theory of Road Pricing. Elsevier Ltd.

Zhou, Z., Chen, A., and Behkor, S., 2012. C-logit stochastic user equilibrium model: Formulations and solution algorithm. Transportmetrica 8(1), 17-41. 
Table 1. Three scenarios of link capacity constraints

\begin{tabular}{c|c|c|c|c|c}
\hline Link & Scenario 1 & Scenario 2 & Scenario 3 & Physical Capacity \\
\cline { 2 - 4 }$(a \in \bar{A})$ & $\begin{array}{c}\text { Threshold } \\
\left(H_{a}^{1}\right)\end{array}$ & $\begin{array}{c}\text { Threshold } \\
\left(H_{a}^{2}\right)\end{array}$ & $\begin{array}{c}\text { Threshold } \\
\left(H_{a}^{3}\right)\end{array}$ & SUE Flows \\
\hline 3 & 15000 & 5000 & 3000 & 25900 & 3383 \\
10 & 4000 & 3500 & 3000 & 4908 & 6953 \\
15 & 4500 & 4000 & 3000 & 4948 & 8819 \\
30 & 4500 & 4000 & 3000 & 4993 & 7148 \\
50 & 12000 & 9000 & 8000 & 19679 & 14507 \\
70 & 4500 & 4000 & 3000 & 5000 & 9205 \\
\hline
\end{tabular}

Table 2. SUE link flows and optimal Lagrangian multipliers

\begin{tabular}{c|cc|cc|cc}
\hline \multirow{2}{*}{$\begin{array}{c}\text { Link } \\
(a \in \bar{A})\end{array}$} & \multicolumn{2}{|c|}{ Scenario 1 } & \multicolumn{2}{c|}{ Scenario 2 } & \multicolumn{2}{c}{ Scenario 3 } \\
\cline { 2 - 7 } & $v_{a}\left(\mathbf{u}^{*}\right) / H_{a}^{1}$ & $u_{a}^{*}$ & $v_{a}\left(\mathbf{u}^{*}\right) / H_{a}^{2}$ & $u_{a}^{*}$ & $v_{a}\left(\mathbf{u}^{*}\right) / H_{a}^{3}$ & $u_{a}^{*}$ \\
\hline 3 & 0.31 & 0.00 & 0.96 & 0.00 & 1.01 & 4.37 \\
10 & 1.00 & 2.95 & 1.00 & 1.87 & 1.00 & 2.76 \\
15 & 1.01 & 4.81 & 1.00 & 5.56 & 1.01 & 7.84 \\
30 & 1.00 & 2.79 & 1.00 & 3.36 & 1.01 & 4.37 \\
50 & 1.00 & 0.93 & 1.00 & 1.82 & 1.00 & 2.57 \\
70 & 1.01 & 4.40 & 1.01 & 3.49 & 1.00 & 4.48 \\
\hline
\end{tabular}

Table 3. Sensitivity test on the demand

\begin{tabular}{c|cc|cc|cc}
\hline \multirow{2}{*}{$\begin{array}{c}\text { Link } \\
(a \in \bar{A})\end{array}$} & \multicolumn{2}{|c|}{$\rho=0.05$} & \multicolumn{2}{c|}{$\rho=0.01$} & \multicolumn{2}{c}{$\rho=0.005$} \\
\cline { 2 - 7 } & $v_{a}\left(\mathbf{u}^{*}\right) / H_{a}^{1}$ & $u_{a}^{*}$ & $v_{a}\left(\mathbf{u}^{*}\right) / H_{a}^{2}$ & $u_{a}^{*}$ & $v_{a}\left(\mathbf{u}^{*}\right) / H_{a}^{3}$ & $u_{a}^{*}$ \\
\hline 3 & 0.14 & 0.00 & 0.31 & 0.00 & 0.36 & 0.00 \\
10 & 1.00 & 0.71 & 1.00 & 2.95 & 1.01 & 3.47 \\
15 & 1.01 & 1.76 & 1.01 & 4.81 & 1.01 & 5.37 \\
30 & 0.97 & 0.00 & 1.00 & 2.79 & 1.01 & 3.54 \\
50 & 0.79 & 0.00 & 1.00 & 0.93 & 1.00 & 1.38 \\
70 & 1.01 & 1.66 & 1.01 & 4.40 & 1.01 & 5.12 \\
\hline
\end{tabular}




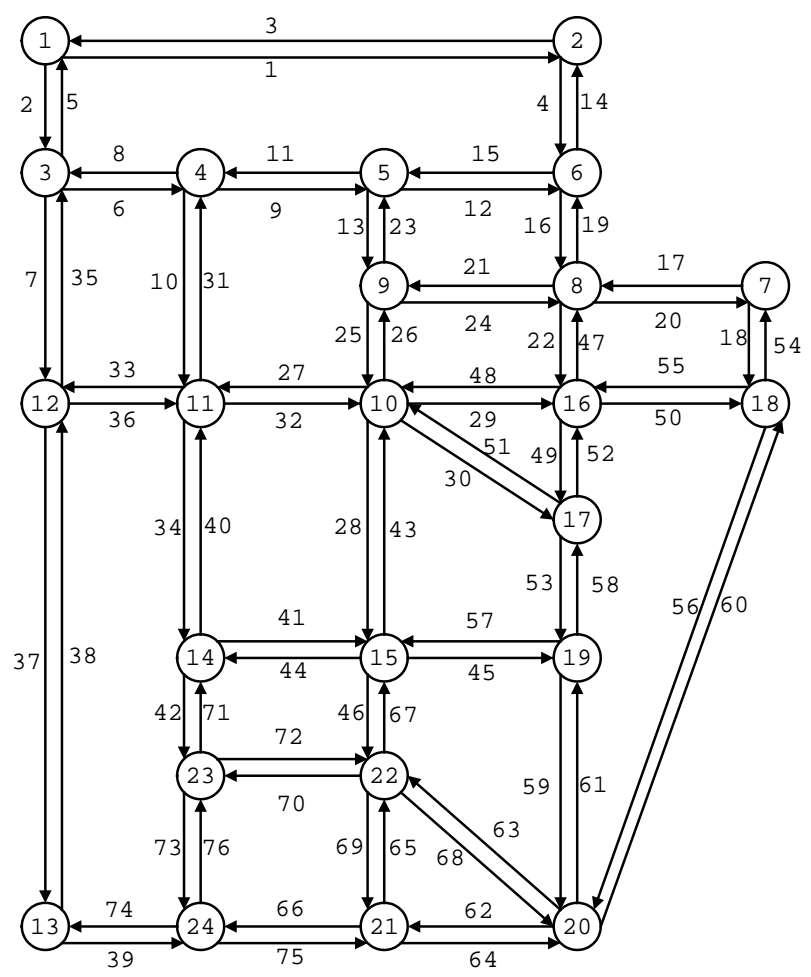

Figure 1. Sioux-Falls Network

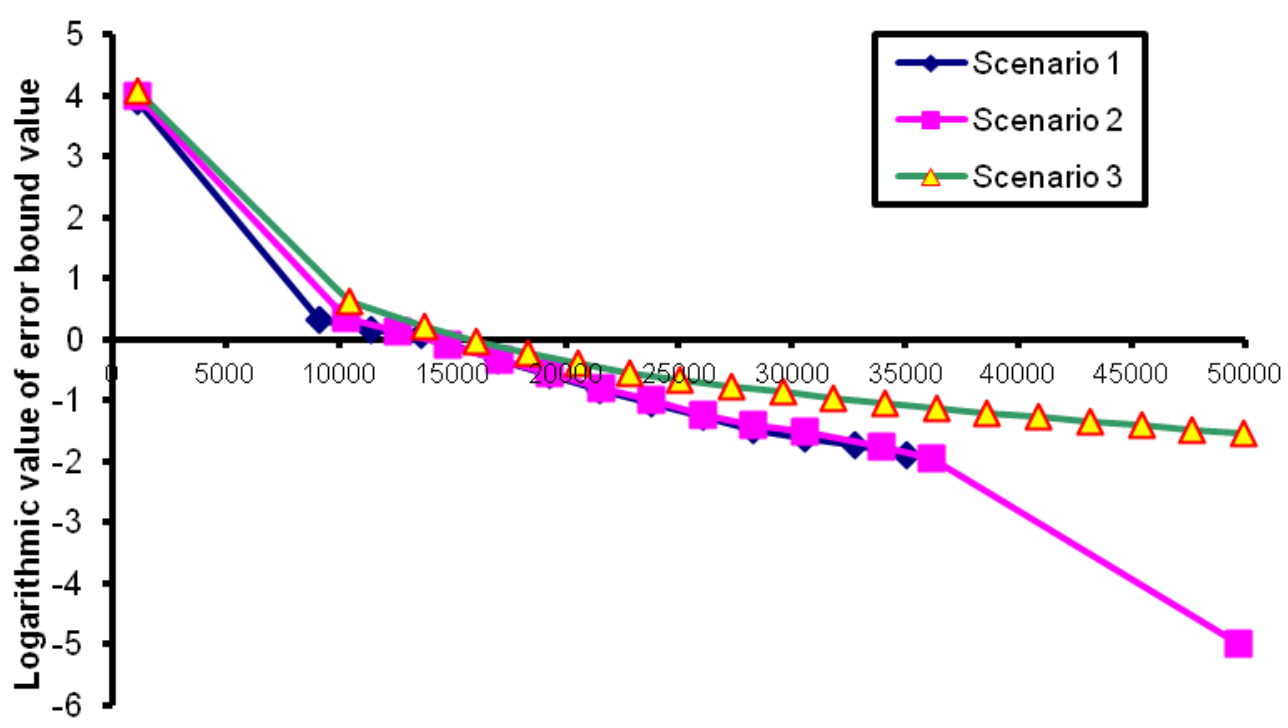

CPU time (seconds)

Figure 2. Convergence trend of the PC algorithm for each scenario 